\title{
Review
}

\section{Electromagnetic scattering by a morphologically complex object: Fundamental concepts and common misconceptions}

\author{
Michael I. Mishchenko ${ }^{\mathrm{a}, *}$, Victor P. Tishkovets ${ }^{\mathrm{b}}$, Larry D. Travis ${ }^{\mathrm{a}}$, Brian Cairns ${ }^{\mathrm{a}}$, \\ Janna M. Dlugach ${ }^{\mathrm{c}}$, Li Liu ${ }^{\mathrm{a}}$, Vera K. Rosenbush ${ }^{\mathrm{c}}$, Nikolai N. Kiselev ${ }^{\mathrm{c}}$ \\ a NASA Goddard Institute for Space Studies, 2880 Broadway, New York, NY 10025, USA \\ ${ }^{\mathrm{b}}$ Institute of Radio Astronomy of NASU, 4 Chervonopraporna Street, Kharkiv 61002, Ukraine \\ ${ }^{\mathrm{c}}$ Main Astronomical Observatory of the National Academy of Sciences of Ukraine, 27 Zabolotny Street, Kyiv 03680, Ukraine
}

\section{A R T I C L E I N F O}

\section{Article history:}

Received 11 January 2010

Received in revised form

2 March 2010

Accepted 29 March 2010

Available online 1 April 2010

\section{Keywords:}

Macroscopic Maxwell equations

Electromagnetic scattering

Multiple scattering

Coherent backscattering

Radiative transfer

\begin{abstract}
A B S T R A C T
Following Keller (Proc Symp Appl Math 1962;13:227-46), we classify all theoretical treatments of electromagnetic scattering by a morphologically complex object into firstprinciple (or "honest" in Keller's terminology) and phenomenological (or "dishonest") categories. This helps us identify, analyze, and dispel several profound misconceptions widespread in the discipline of electromagnetic scattering by solitary particles and discrete random media. Our goal is not to call for a complete renunciation of phenomenological approaches but rather to encourage a critical and careful evaluation of their actual origin, virtues, and limitations. In other words, we do not intend to deter creative thinking in terms of phenomenological short-cuts, but we do want to raise awareness when we stray (often for practical reasons) from the fundamentals. The main results and conclusions are illustrated by numerically-exact data based on direct numerical solutions of the macroscopic Maxwell equations.
\end{abstract}

Published by Elsevier Ltd.

\section{Contents}

1. Introduction: microphysical and phenomenological approaches to electromagnetic scattering $\ldots \ldots \ldots \ldots \ldots \ldots \ldots \ldots . \ldots 6$

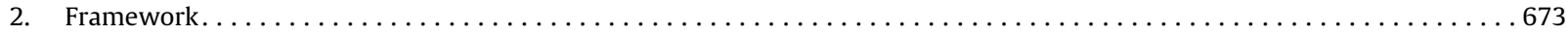

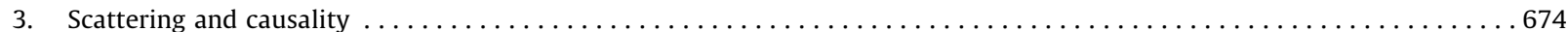

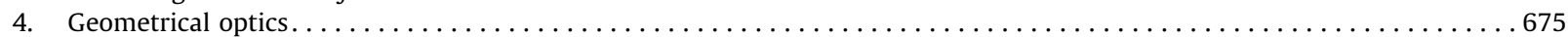

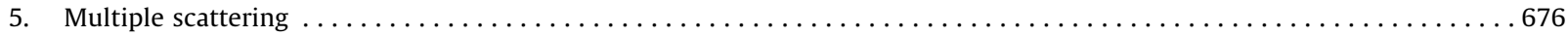

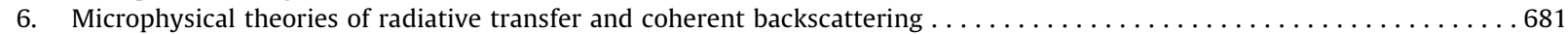

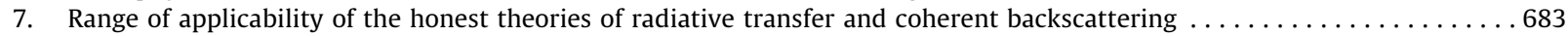

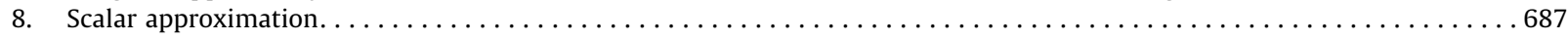

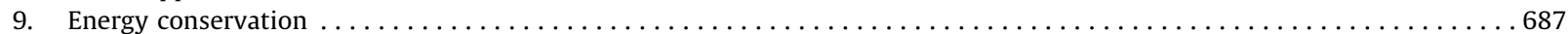

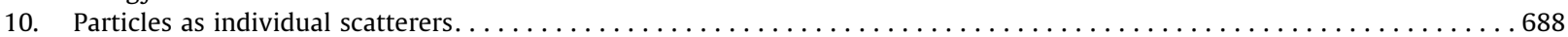

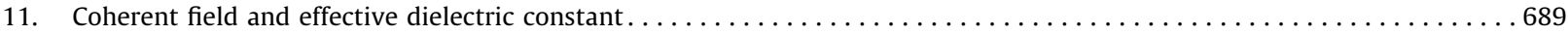

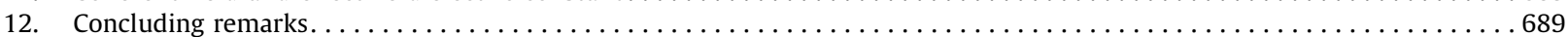

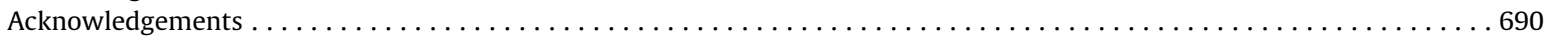

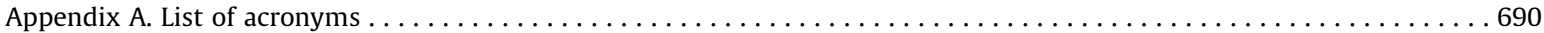

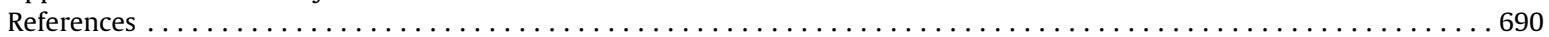

\footnotetext{
* Corresponding author. Fax: +12126785622.

E-mail addresses: crmim2@gmail.com, mmishchenko@giss.nasa.gov (M.I. Mishchenko).
} 


\section{Introduction: microphysical and phenomenological approaches to electromagnetic scattering}

Scientific, biomedical, and engineering problems involving the scattering of light (or other electromagnetic radiation) by a morphologically complex macroscopic object are quite common. Among typical examples of a complex scattering object are a cloud, a particulate surface, a particle suspension, a tissue sample, or an isolated morphologically complex particle. Quite often electromagnetic scattering by a complex object is addressed without an explicit solution of the macroscopic Maxwell equations (MMEs). In some cases it is acknowledged that the MMEs do control the scattering phenomenon, but then it is claimed that a direct solution of the MMEs is far too difficult to attempt. In many cases the MMEs are not mentioned at all. Instead, an ad hoc "approximation" is used and is essentially elevated to the level of an independent basic physical principle, as exemplified by the phenomenological radiative transfer theory (RTT). Usually this is done based on vague "physical grounds", which is a traditional implicit excuse for not being able to derive the desired outcome mathematically from primordial equations such as the MMEs.

Such approximations are often based on "physical concepts" many of which are the consequence of trying to describe a complex physical phenomenon using a simplified analogy. For example, the propagation of electromagnetic waves is often described as being analogous to the propagation of waves on the surface of a pond. Such an analogy may serve to increase the level of mental comfort of students by helping them "visualize" a physical phenomenon that escapes completely human natural senses. However, this analogy can be quite misleading and contains no real physics whatsoever since electromagnetic waves are not mechanical surface waves. Instead, real physics is contained in the proper selection of mathematical equations intended to adequately describe specific natural phenomena. Once these primordial equations have been formulated, solving these equations directly without invoking any ad hoc "physical concepts" would solve all real needs of the physicist.

Let us imagine, for example, that we have at our disposal a direct computer solver of the MMEs (in the form of a suitable \{computer; computer program\} combination) applicable to an arbitrarily complex object. Then we would not need any approximation and any physical concept not already contained in the MMEs in order to interpret laboratory or remote-sensing measurements of electromagnetic scattering. Indeed, the output of any measurement could then be modeled by solving the MMEs once for a fixed object or many times for a representative set of realizations of a random object (such as a cloud) supplemented by statistical averaging of the relevant optical observables.

Unfortunately, a direct solver of the MMEs applicable to a real cloud of liquid water droplets or ice crystals does not exist and is unlikely to become available in the near future. Hence the widespread use of "approximate" treatments of electromagnetic scattering by complex macroscopic objects.
Paraphrasing Keller [1] and using his terminology, all theoretical methods for treating electromagnetic scattering by a morphologically complex object can be classified into two categories: "honest" (or microphysical) and "dishonest" (or phenomenological). ${ }^{1}$ An honest method is the result of solving the MMEs, perhaps after making one or more specific and well defined assumptions intended to simplify the solution. For example, the Rayleigh approximation [2] is the result of solving the MMEs under the assumption that the product of the wave number and the maximal particle dimension is much smaller than unity, while the Fresnel formulas and coefficients follow from the assumption that a plane wave is incident on a perfectly flat interface separating two homogeneous half-spaces with different real-valued refractive indices. The practical applicability of a microphysical method usually requires no validation provided that all underlying assumptions are indeed satisfied. However, if an honest method is used to model situations in which one or more of the underlying assumptions are violated then the quantitative applicability of this approach must be carefully examined [3].

Fundamentally, an honest method is by definition the result of an explicit direct solution of the MMEs, e.g., a closed-form analytical solution or a numerically-exact computer solution. The former is often the consequence of taking an asymptotic limit (e.g., assuming that the product of the wave number and the distance from the scattering object to the observation point is much greater than unity, which renders the far-field approximation). The latter is the outcome of running a direct computer solver of the MMEs generating numbers with a guaranteed number of correct decimals. The number of correct decimals may vary depending on the available computer resources and practical accuracy requirements. However, all reported decimals can, in principle, be validated by modifying computer program settings in order to accommodate a more stringent accuracy requirement.

Quite often the use of a microphysical analytical method allows one to identify certain idealized physical concepts. Typical examples would be the asymptotic short-wave concept of a light ray propagating in a continuous medium, the concepts of reflection and refraction of waves by a plane interface, the concepts of wave interference and diffraction, and the concept of farfield scattering. Such concepts are unnecessary in principle and are nothing more than verbal characterizations of formulas derived from the MMEs. However, they constitute what is usually called "physical understanding of the problem" and as such may have some positive heuristic value and facilitate qualitative "interpretation" of formulas, digital computer outputs, or experimental data,

\footnotetext{
${ }^{1}$ Of course, the words "honest" and "dishonest" are intended to characterize methods rather than human character traits. However, the terms "honest" and "dishonest theoretical methods" can be viewed by some as having the connotation of a moral judgment about the practitioners of such methods. Minding those who believe that Keller's terminology may be excessively figurative, we will often use the words "microphysical" and "phenomenological" as substitutes for "honest" and "dishonest", respectively.
} 
wherein formulas and numbers are "explained" or "interpreted" by using words. However, it is very important to remember that such derivative concepts represent idealizations, cannot replace the original mathematical formulas, and should not be used outside the range of their validity defined by the explicit solution of the MMEs.

A phenomenological approach to electromagnetic scattering is not based on an explicit solution of the MMEs for the specific scattering object in question. Instead, it is a conglomerate of formulas and concepts borrowed, on an ad hoc basis, from various honest (or even other phenomenological) approaches. Almost always a phenomenological approach is "derived" by saying words under the pretense that these words represent a "physical understanding of the problem" which, presumably, allows one to bypass writing down the MMEs and solving them mathematically.

A classical example of a phenomenological approach is the geometrical theory of diffraction (GTD) of electromagnetic waves in inhomogeneous media [4,5]. In fact, the book by Borovikov and Kinber [4] is quite remarkable in that it states explicitly that the GTD is based on a set of "postulates" intended to define the "directions of diffracted rays". In other words, it is recognized that the "intuitively obvious" concept of rays is incompatible with the physical phenomenon of diffraction of waves, and so ad hoc postulates (i.e., patches) not following directly from the MMEs are necessary to combine these motley concepts into a practical computational procedure.

Unfortunately, such honest accounts of phenomenological approaches are quite rare. Usually it is not recognized that a verbally stated "physical understanding" represents, in fact, one or several postulates which implicitly supplement or even replace the MMEs. It is, however, a wellknown mathematical fact that a properly formulated combination of the MMEs and boundary conditions has a solution, this solution being unique. This means that any additional postulate is unnecessary at best and can contradict the MMEs at worst. As a consequence, phenomenological approaches often serve as a plentiful source of misconceptions hindering further progress in the theory of electromagnetic scattering and its practical applications.

The main objective of this essay is to identify, analyze, and dispel several such misconceptions widespread in the discipline of electromagnetic scattering by an isolated particle or a discrete random medium (DRM). To paraphrase Keller [1] once more, our goal is not to call for a complete renunciation of phenomenological approaches but rather to encourage a critical and open evaluation of their actual origin, virtues, and limitations. It is hoped that in certain cases this can result in an improved justification of a phenomenological method by showing that its results are, in some cases, useful quantitative approximations to a microphysical solution.

\section{Framework}

In what follows, we will assume that the interaction of electromagnetic radiation with an isolated particle or a
DRM is fully described by the MMEs supplemented by appropriate boundary conditions [6-8]. In other words, it is assumed that at each moment in time, the entire scattering object can be represented by a specific spatial configuration of a number $N \geq 1$ of discrete finite particles (Fig. 1). A solitary particle $(N=1)$ or any constituent particle of a group $(N>1)$ is assumed to be sufficiently large so that it can be characterized by optical constants appropriate to bulk matter. In terms of classical macroscopic electromagnetics, the presence of a particle means that the optical constants inside the particle volume are different from those of the surrounding host medium. The spatial distribution of the optical constants throughout the scattering object defines the corresponding boundary conditions which, along with the radiation condition at infinity $[9,10]$, make the solution of the MMEs unique.

A more fundamental way to treat electromagnetic scattering by the object shown in Fig. 1 would be to consider it as a vast collection of elementary particles and use the formalism of quantum electrodynamics (QED). We do not do that for two reasons. First of all, the direct application of this approach is impracticable, given the enormous complexity of the scattering object at the elementary-particle level, and, to the best of our knowledge, has never been attempted. Second of all, it is known that by combining the concepts of QED and statistical mechanics and assuming that the scattering object is macroscopic, one can arrive at the classical MMEs [11,12]. This means that certain optical observables can be computed with sufficient accuracy by solving the MMEs, which allows us to adopt them as primordial physical equations in the specific context of our analysis of electromagnetic scattering. Importantly, by virtue of having their origin in QED, the MMEs themselves represent an honest approach to the quantitative description of a wide range of electromagnetic phenomena.

Another basic assumption will be that all fields and sources vary in time according to the complex-exponential time dependence $\exp (-\mathrm{i} \omega t)$, where $t$ is time, $\omega$ is the angular frequency, and $\mathrm{i}=(-1)^{1 / 2}$. In other words, our analysis will be explicitly limited to frequency-domain electromagnetic scattering $[6,9,10,13,14]$. For the sake of brevity, the factor $\exp (-\mathrm{i} \omega t)$ will thereafter be omitted.

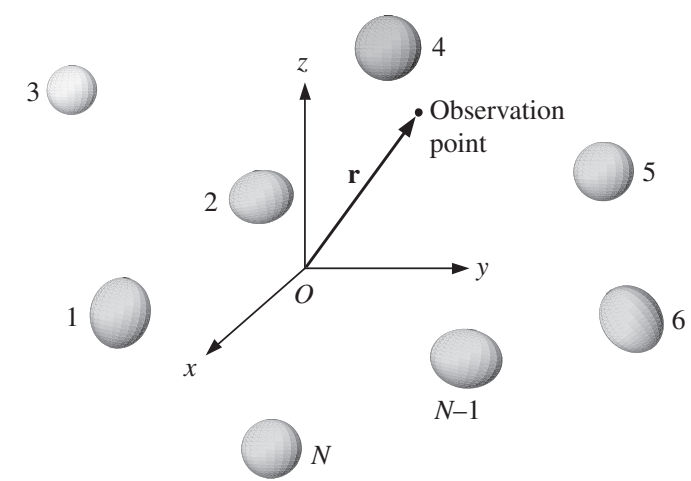

Fig. 1. Scattering object in the form of a group of $N$ discrete particles. 
It is important to recognize that QED is based on the quantization of the microscopic electromagnetic field interacting with elementary particles and involves photons, each photon being a quantum of a single normal mode of the microscopic field [15-21]. As such, photons are neither waves nor localized particles of light [15-23]. During the explicit derivation of the MMEs from QED, photons and elementary particles "disappear", so that the MMEs involve only the macroscopic electromagnetic field and macroscopic optical constants. Therefore, if one wants to invoke the concept of photons to describe electromagnetic scattering by a macroscopic object, the proper way to do that is to retract the MMEs and consistently use the equations and concepts of QED to describe the interaction of the group of elementary particles constituting the scatterer with the microscopic electromagnetic field. ${ }^{2}$ Otherwise juggling with "photons" and "waves" at will and ascribing to the "photons" desirable rather than actual properties results in the notorious "photonic confusion" discussed in detail in $[7,8,25]$.

\section{Scattering and causality}

Perhaps the most profound misconception in the discipline of electromagnetic scattering is that scattering by an isolated particle is a solitary process unfolding in time continuously wherein the incident field becomes transformed into the scattered field (see [25] for a more extensive discussion). This process allegedly involves a preceding incoming wave, the physical interaction of this wave with the particle, and the subsequent transformation of the incident wave into the outgoing scattered wave. Furthermore, the incident wave is often portrayed as the physical cause of the scattered wave.

However, the actual way to define electromagnetic scattering is to solve the MMEs twice. The first solution, in terms of the respective pair of the electric and magnetic fields $\left\{\mathbf{E}_{1}(\mathbf{r}), \mathbf{H}_{1}(\mathbf{r})\right\}$, corresponds to the situation with no scattering object, whereas the second solution, $\left\{\mathbf{E}_{2}(\mathbf{r}), \mathbf{H}_{2}(\mathbf{r})\right\}$, corresponds to the situation with a scattering object present, where $\mathbf{r}$ is the position vector of the observation point (Fig. 1). The second solution is intentionally sought in the form

$\mathbf{E}_{2}(\mathbf{r})=\mathbf{E}_{1}(\mathbf{r})+\mathbf{E}_{3}(\mathbf{r})$,

$\mathbf{H}_{2}(\mathbf{r})=\mathbf{H}_{1}(\mathbf{r})+\mathbf{H}_{3}(\mathbf{r})$,

where the vector fields $\mathbf{E}_{3}(\mathbf{r})$ and $\mathbf{H}_{3}(\mathbf{r})$ are required to satisfy the radiation condition at infinity $[9,10]$ by decaying as the inverse distance from the object. The total field in the absence of the particle is called the incident field,

\footnotetext{
${ }^{2}$ Note in this regard that the so-called "cavity QED" based on the quantization of the macroscopic electromagnetic field remains a phenomenological theory [24]. In fact, this theory can hardly be selfconsistent in principle since the MMEs are derived by quantizing the microscopic electromagnetic field in the first place. The legitimacy of a repeated quantization of the resulting macroscopic electromagnetic field is quite questionable and does not follow from any fundamental physical principles. As a consequence, the cavity QED and similar approaches based on the quantization of the macroscopic electromagnetic field remain "dishonest" theories.
}

whereas the difference between the total field in the presence of the particle and the total field that would exist in the absence of the particle is called the scattered field:

$\mathbf{E}^{\mathrm{sca}}(\mathbf{r}) \equiv \mathbf{E}_{3}(\mathbf{r})=\mathbf{E}_{2}(\mathbf{r})-\mathbf{E}_{1}(\mathbf{r})=\mathbf{E}_{2}(\mathbf{r})-\mathbf{E}^{\mathrm{inc}}(\mathbf{r})$,

$\mathbf{H}^{\mathrm{sca}}(\mathbf{r}) \equiv \mathbf{H}_{3}(\mathbf{r})=\mathbf{H}_{2}(\mathbf{r})-\mathbf{H}_{1}(\mathbf{r})=\mathbf{H}_{2}(\mathbf{r})-\mathbf{H}^{\text {inc }}(\mathbf{r})$.

Thus, it is the modification of the total electromagnetic field resulting from the presence of the particle that is called electromagnetic scattering. This means that although electromagnetic scattering can be said to be a physical phenomenon (amounting to the fact that the total fields computed in the presence and in the absence of a particle are different), it is not a solitary physical process.

Eqs. (3) and (4) demonstrate that the total field in the presence of the particle is intentionally represented as the sum of the incident and scattered fields:

$\mathbf{E}(\mathbf{r}) \equiv \mathbf{E}_{2}(\mathbf{r})=\mathbf{E}^{\mathrm{inc}}(\mathbf{r})+\mathbf{E}^{\mathrm{sca}}(\mathbf{r})$,

$\mathbf{H}(\mathbf{r}) \equiv \mathbf{H}_{2}(\mathbf{r})=\mathbf{H}^{\text {inc }}(\mathbf{r})+\mathbf{H}^{\text {sca }}(\mathbf{r})$,

where $\mathbf{E}^{\text {inc }}(\mathbf{r})$ and $\mathbf{H}^{\text {inc }}(\mathbf{r})$ are obtained by solving the MMEs in the absence of the particle. This makes both the incident and the scattered field, as they appear in Eqs. (5) and (6), purely mathematical quantities. This implies, in particular, that

- the incident field is not modified by scattering and is not transformed into the scattered field;

- the purely mathematical incident field cannot interact physically with the particle;

- the purely mathematical incident field cannot be a physical cause of the purely mathematical scattered field;

- only the total field, either in the absence or in the presence of the particle, is a real physical field.

These points are well exemplified by the so-called volume integral equation (VIE), which follows directly from the MMEs. The VIE for the electric field reads

$$
\begin{aligned}
\mathbf{E}(\mathbf{r})= & \mathbf{E}^{\mathrm{inc}}(\mathbf{r})+k_{1}^{2} \int_{V_{\mathrm{INT}}} \mathrm{d} \mathbf{r}^{\prime} \stackrel{\leftrightarrow}{G}\left(\mathbf{r}, \mathbf{r}^{\prime}\right) \cdot \mathbf{E}\left(\mathbf{r}^{\prime}\right)\left[m^{2}\left(\mathbf{r}^{\prime}\right)-1\right] \\
= & \mathbf{E}^{\mathrm{inc}}(\mathbf{r})+k_{1}^{2}\left(\stackrel{\leftrightarrow}{I}+\frac{1}{k_{1}^{2}} \nabla \otimes \nabla\right) \\
& \cdot \int_{V_{\mathrm{INT}}} \mathrm{d} \mathbf{r}^{\prime} \mathbf{E}\left(\mathbf{r}^{\prime}\right) \frac{\exp \left(\mathrm{i} k_{1}\left|\mathbf{r}-\mathbf{r}^{\prime}\right|\right)}{4 \pi\left|\mathbf{r}-\mathbf{r}^{\prime}\right|}\left[m^{2}\left(\mathbf{r}^{\prime}\right)-1\right]
\end{aligned}
$$

$$
\mathbf{r} \in \mathfrak{R}^{3},
$$

where $m\left(\mathbf{r}^{\prime}\right)$ is the refractive index of the particle interior relative to that of the host exterior medium, $k_{1}$ is the wave number in the host medium, $\leftrightarrow\left(\mathbf{G}, \mathbf{r}^{\prime}\right)$ is the free space dyadic Green's function, $\stackrel{I}{I}$ is the identity dyadic, $\otimes$ is the dyadic product sign, $V_{\text {INT }}$ is the interior volume of the particle, and $\mathfrak{R}^{3}$ is the entire three-dimensional space. One can see that the VIE expresses the total field 
everywhere in space in terms of the total field inside the scattering object. ${ }^{3}$ If the scattering object is absent then $m\left(\mathbf{r}^{\prime}\right) \equiv 1$, and the total field is identically equal to the incident field. Otherwise the total field can be represented mathematically as a vector sum of the incident and scattered components, the latter being given by the second term on the right-hand side of Eq. (7).

Eq. (7) makes it quite obvious that the particle interacts with the total field rather than with the incident field. Therefore, if one wants to identify the cause of the scattered field then it is the very presence of the particle. This conclusion is quite consistent with the above definition of the phenomenon of electromagnetic scattering.

The erroneous characterization of electromagnetic scattering by a particle as a solitary physical process unfolding in time undoubtedly stems from the abovementioned "photonic confusion" (e.g., "a photon flies up to a macroscopic object and bounces off of it, thereby creating the scattered field") as well as from the misuse of geometrical optics (e.g., "a ray impinges on the surface of the particle and gets partly reflected from and partly refracted into the particle; the reflected rays and the rays refracted outside, perhaps after two or more internal reflections, create the scattered field"). Such characterizations of electromagnetic scattering fall apart as soon as one begins to scrutinize the exact definitions and physical meaning of the specific terms involved (e.g., [25]).

The verbally formulated causality of electromagnetic scattering has been used to derive the so-called sum rule for the extinction cross section by expressing the integral of the extinction cross section over all wavelengths in terms of a simple product of the particle volume and a coefficient depending on the particle shape and static dielectric function [27]. Several other sum rules have been derived, again using the "causality" consideration (see, e.g., references in [28]). However, as discussed above, the scattered field is not caused by the incident field. Therefore, alleged mathematical incarnations of the erroneously stated "physical" causality must be derived directly from the MMEs rather than accepted as "physically obvious" facts. Otherwise, they represent additional postulates which, in fact, may contradict the MMEs. Therefore, the resulting sum rules should be considered unproven hypotheses rather than outcomes of rigorous derivations from first principles [28].

\section{Geometrical optics}

An instructive example of a phenomenological theoretical approach is the geometrical optics method (GOM) widely used to describe far-field electromagnetic scattering by a nonspherical particle $[29,30]$. The GOM is often characterized as an asymptotic solution of the MMEs in the limit $k_{1} D_{\min } \rightarrow \infty$, where $D_{\min }$ is the smallest

\footnotetext{
${ }^{3}$ Note that writing the VIE in the form of the first line of Eq. (7) implies that the singularity of the dyadic Green's function is treated in an appropriate way (see [26] and references therein). This comment also applies to Eq. (14) below.
}

dimension of the particle, although this characterization is generally incorrect. In the case of scattering by a perfect homogeneous sphere, many features of the GOM can be justified, at least partially, by studying the asymptotic behavior of the Lorenz-Mie coefficients [31-35]. However, the availability and great numerical efficiency of the exact Lorenz-Mie theory limit the practical usefulness of the GOM in the particular case of spherical particles. The GOM is most useful in application to nonspherical particles, since all numerically-exact techniques become quite time-consuming, if even applicable, when the particle size becomes much greater than the wavelength [36-38]. In this general case, however, the GOM has never been derived directly from the MMEs by evaluating the limit $k_{1} D_{\text {min }} \rightarrow \infty$. Instead, it remains a collection of ad hoc formulas, recipes, and concepts united by "simple physical considerations". This does not allow one to characterize the GOM as an honest technique. Given the complexity of the general scattering problem, this situation is unlikely to change in the future.

Besides the simplest version of the GOM combining the Snell-Fresnel ray tracing with diffraction on the particle projection, several more sophisticated versions of the GOM have been proposed, such as the GTD and the socalled Kirchhoff approximation (see, e.g., [4,5,29,39-41] and references therein). Like with any phenomenological approach, the range of applicability of these and similar techniques is not well defined, and their quantitative applicability should be extensively examined versus numerically-exact results [41-47].

As an example, Fig. 2 depicts the results of GOM and Lorenz-Mie computations of the phase function $a_{1}(\Theta)$ for nonabsorbing homogeneous spheres [36,48], where $\Theta \in$ $[0, \pi]$ is the scattering angle defined as the angle between the incidence and scattering directions. The phase function describes the angular distribution of scattered light for unpolarized incident intensity and is normalized according to

$\frac{1}{2} \int_{0}^{\pi} \mathrm{d} \Theta \sin \Theta a_{1}(\Theta)=1$

The Lorenz-Mie results are averaged over a narrow size distribution with an effective variance of 0.07 , so that $x$ represents the effective size parameter $k_{1} r_{\text {eff }}, r_{\text {eff }}$ being the effective radius of the size distribution [48]. The GOM results are computed for a single sphere with a size parameter $x=k_{1} a$, where $a$ is the sphere radius. The raytracing and diffraction components of the GOM phase functions have been averaged over $1^{\circ}$-wide angular bins. One can see that the GOM phase-function results for spheres become reasonably accurate only at size-parameter values exceeding several hundred. Furthermore, the simple GOM is unable to reproduce the strong enhancement of intensity in the backscattering direction traditionally called the glory. However, the indisputable heuristic value of the GOM is the qualitative prediction of the local maxima at scattering angles close to $137^{\circ}$ and $130^{\circ}$ called the primary and secondary rainbows.

Fig. 3 shows the results of approximate GOM and numerically-exact $T$-matrix computations of the phase 


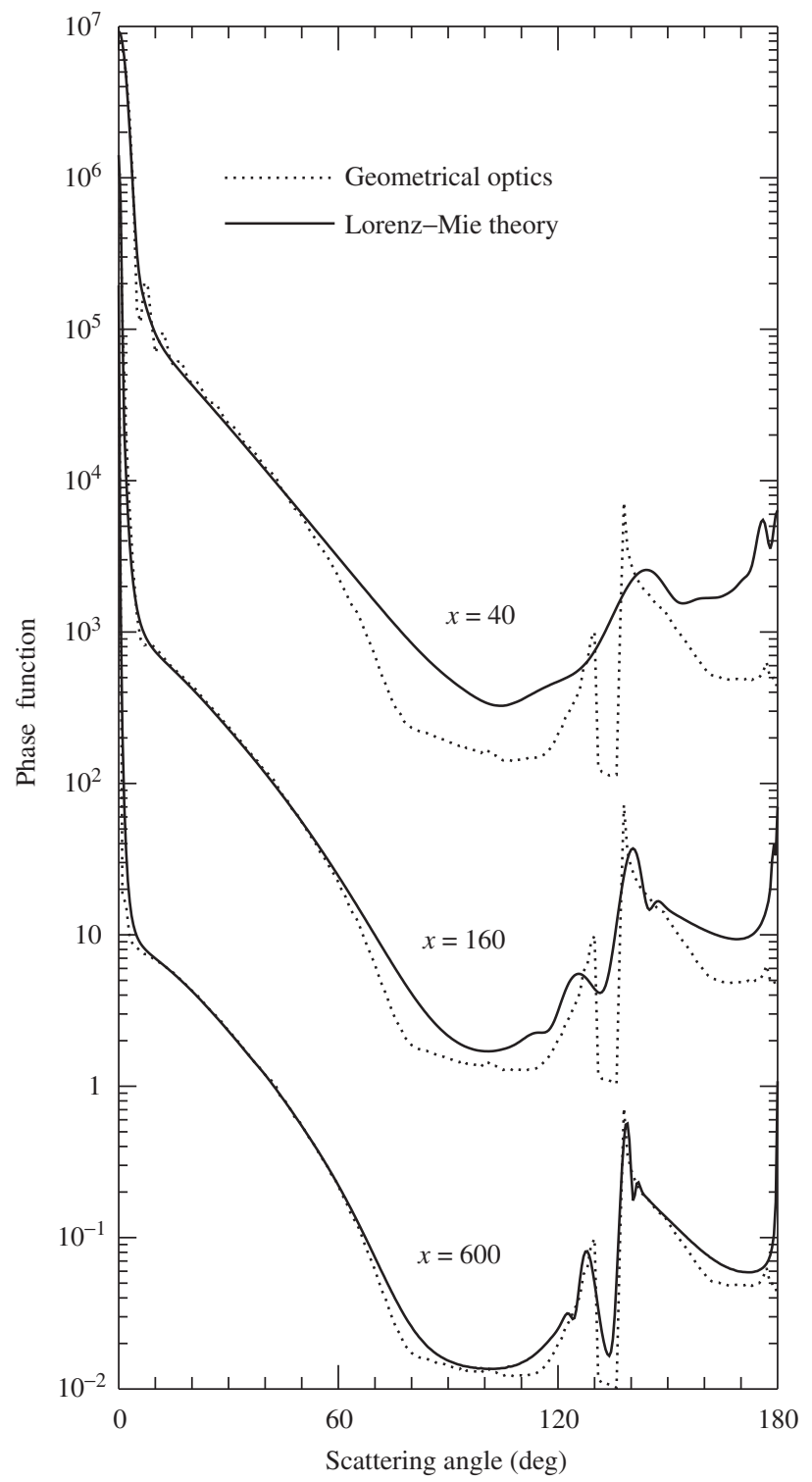

Fig. 2. Phase function versus scattering angle computed with the GOM and Lorenz-Mie theory for homogeneous spheres with a relative refractive index $m=1.33$ and size parameters $x=40,160$, and 600 . The vertical axis scale applies to the curves with $x=600$, the other curves being successively displaced upward by a factor of 100 .

function for monodisperse, randomly oriented circular cylinders with a diameter-to-length ratio of unity and a relative refractive index $m=1.311$ [36,44]. The quasiperiodic ripple in the $T$-matrix curves is caused by interference effects characteristic of monodisperse particles, the scattering-angle frequency of this ripple being inversely proportional to particle size parameter (see the discussion in the following section). Contrasting Figs. 2 and 3 appears to suggest that GOM results for nonspherical particles in random orientation may be somewhat more accurate for a given size parameter than those for surface-equivalent spheres. Again, the heuristic value of the GOM in this case is its ability to predict qualitatively specific scattering-angle features such as the $46^{\circ}$ halo and the strong and narrow retroreflection peak centered at the exact backscattering direction.

\section{Multiple scattering}

Throughout scientific literature, one can encounter various definitions of "multiple scattering", all of them stemming from an intuitive idea of successive scattering events caused by a sequence of particles in a multiparticle group. However, the characterization of a morphologically complex object, such as a cloud, as being composed of separate particles is based solely on the 


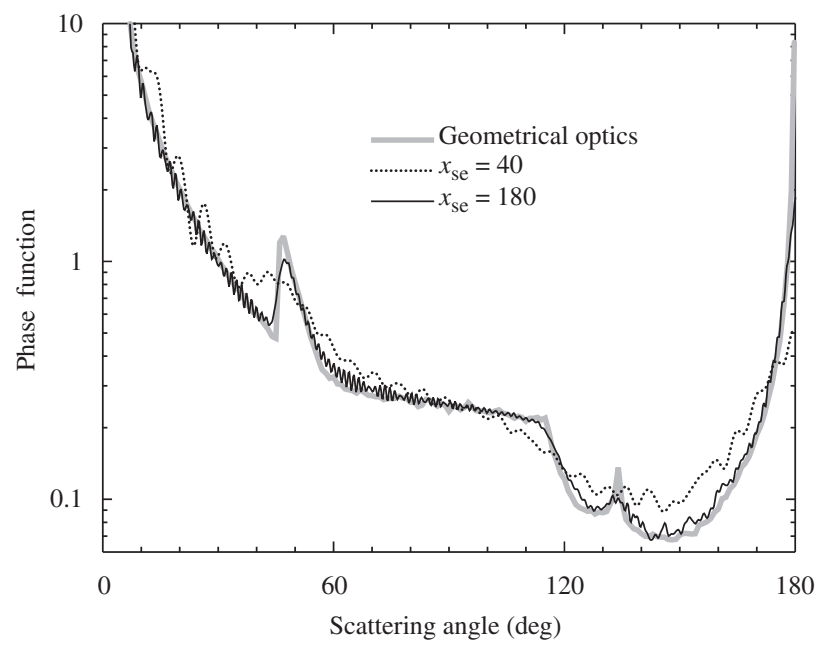

Fig. 3. GOM and $T$-matrix phase functions for monodisperse, randomly oriented circular cylinders with surface-equivalent-sphere size parameters $x_{\mathrm{se}}=40$ and 180 .

human visual perception and is immaterial in the framework of the MMEs. Indeed, the same VIE describes electromagnetic scattering by a "multi-particle system" shown in Fig. 1 as well as by a "single isolated particle", even though the interior volume $V_{\text {INT }}$ in the former case is the union of the "constituent-particle volumes". In other words, an object remains a single, unified scatterer irrespective of the complexity of its morphology.

To illustrate this point, let us consider far-field scattering of a plane electromagnetic wave by three fixed objects shown in Figs. $4 a-c$ : an oblate spheroid with an aspect ratio of 2 , a circular cylinder with a diameter-tolength ratio of one, and a cluster of 80 small identical spherical particles randomly distributed throughout an imaginary spherical volume $V$ having a radius $A$. The volume-equivalent-sphere size parameters of the spheroid and the cylinder are the same and are equal to that of the spherical volume: $k_{1} A=40$. The size parameter of the constituent spherical particles is $k_{1} a=4$. The relative refractive indices of the spheroid, the cylinder, and the constituent spherical particles are the same and are equal to 1.32. The coordinates of the constituent spheres populating the volume $V$ were selected using a random number generator, but otherwise they are fixed.

The laboratory spherical coordinate system used to describe far-field electromagnetic scattering is shown in Fig. $4 d$, in which the unit vectors $\hat{\mathbf{n}}^{\text {inc }}$ and $\hat{\mathbf{n}}^{\text {sca }}$ specify the directions of incidence and scattering, respectively. The orientation of a scattering object with respect to the laboratory frame is specified by the Euler angles of rotation, as shown in Fig. 5 [36]. The corresponding sets of the Euler angles $\{\alpha, \beta, \gamma\}$ are given by $\left\{145^{\circ}, 52^{\circ}, 0^{\circ}\right\}$ for the spheroid and the cylinder and by $\left\{0^{\circ}, 0^{\circ}, 0^{\circ}\right\}$ for the spherical volume.

The angular distribution and polarization state of the scattered light in the far-field zone of the entire scattering object is fully described by the so-called Stokes phase matrix $\mathbf{Z}$ [36]. The latter expresses the Stokes parameters of the light scattered in the observation direction $\hat{\mathbf{n}}^{\mathrm{sca}}$ in terms of those of the incident light:

$\left[\begin{array}{c}I^{\text {sca }} \\ Q^{\text {sca }} \\ U^{\text {sca }} \\ V^{\text {sca }}\end{array}\right] \propto \mathbf{Z}\left(\hat{\mathbf{n}}^{\text {sca }}, \hat{\mathbf{n}}^{\text {inc }}\right)\left[\begin{array}{c}I^{\text {inc }} \\ Q^{\text {inc }} \\ U^{\text {inc }} \\ V^{\text {inc }}\end{array}\right]$.

The Stokes parameters of the incident and scattered light are defined with respect to the corresponding meridional planes. The zenith and azimuth angles of the incidence direction are assumed to be $\theta^{\text {inc }}=0^{\circ}$ and $\varphi^{\text {inc }}=0^{\circ}$ which implies that the meridional plane of the incidence direction coincides with the $x z$ half-plane with $x \geq 0$. The incident light is taken to be circularly polarized in the counter-clockwise sense when looking in the direction of propagation, which implies that $V^{\text {inc }}=I^{\text {inc }}$ and $Q^{\text {inc }}=U^{\text {inc }}=0$.

The left-hand panels of Figs. 6a-c show the far-field angular distributions of the total intensity, $I^{\text {sca }}$, scattered in the backward hemisphere by the three fixed objects. These intensity distributions were computed using the numerically-exact $T$-matrix programs described in [36,49] and demonstrate typical speckle patterns of comparable complexity. The origin of the speckles can be explained qualitatively as follows.

Using the VIE (7), it is rather straightforward to show that sufficiently far from the entire scattering object, the scattered field is given by the following formula of the farfield approximation [36]:

$$
\begin{aligned}
\mathbf{E}^{\mathrm{sca}}(\mathbf{r}) \underset{r \rightarrow \infty}{=} & \frac{\exp \left(\mathrm{i} k_{1} r\right)}{r} \frac{k_{1}^{2}}{4 \pi}\left(\stackrel{\leftrightarrow}{I}-\hat{\mathbf{n}}^{\mathrm{sca}} \otimes \hat{\mathbf{n}}^{\mathrm{sca}}\right) \cdot \int_{V_{\mathrm{INT}}} \mathrm{d} \mathbf{r}^{\prime}\left[m^{2}\left(\mathbf{r}^{\prime}\right)-1\right] \\
& \times \mathbf{E}\left(\mathbf{r}^{\prime}\right) \exp \left(-\mathrm{i} k_{1} \hat{\mathbf{n}}^{\mathrm{sca}} \cdot \mathbf{r}^{\prime}\right),
\end{aligned}
$$

where $r$ is the distance from the origin of the laboratory coordinate system to the observation point. The cause of 
a

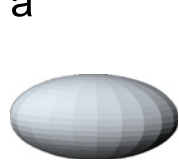

d

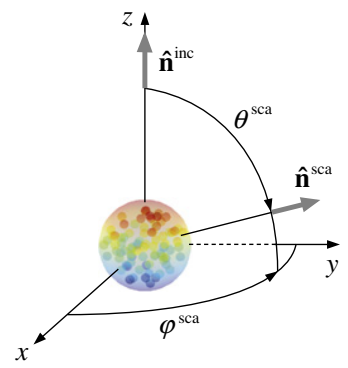

g
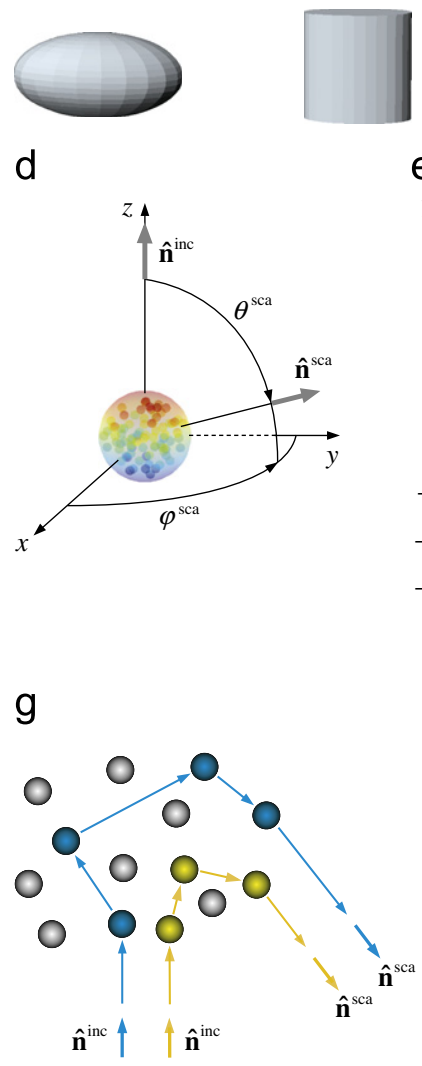

e b

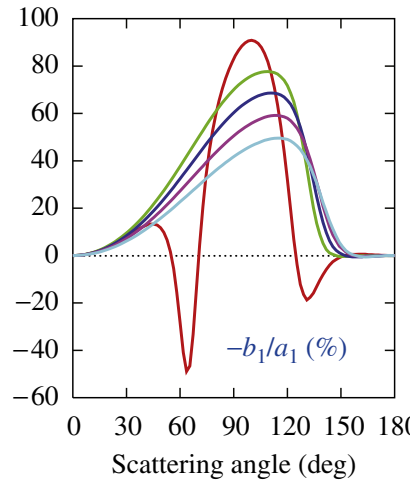

$\mathrm{h}$

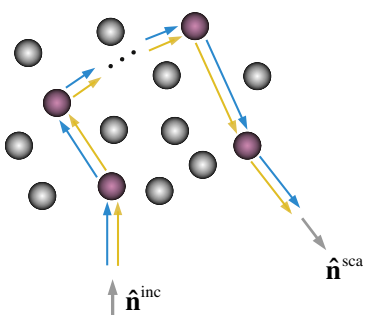

C

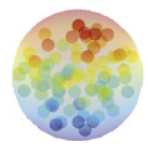

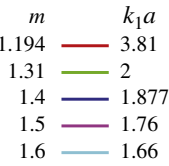

i

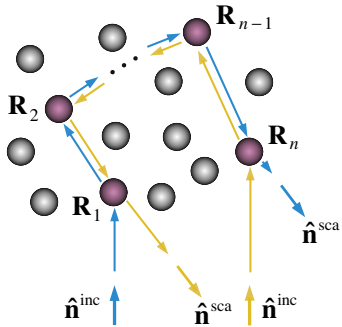

Fig. 4. (a-c) Three scattering objects. (d) Geometry of electromagnetic scattering. (e) Polarization of scattered light for unpolarized incident light (in percent) vs. scattering angle for a homogeneous spherical particle with several \{refractive index, size parameter\} combinations. (f) Forward-scattering interference. (g) Multi-particle wave paths going through different strings of particles. (h) A ladder diagram. (i) A cyclical diagram. For simplicity, the observation point in panels $(\mathrm{g}-\mathrm{i})$ is assumed to be located in the far-field zone of the entire scattering object in the direction $\hat{\mathbf{r}}=\hat{\mathbf{n}}^{\text {sca }}$.

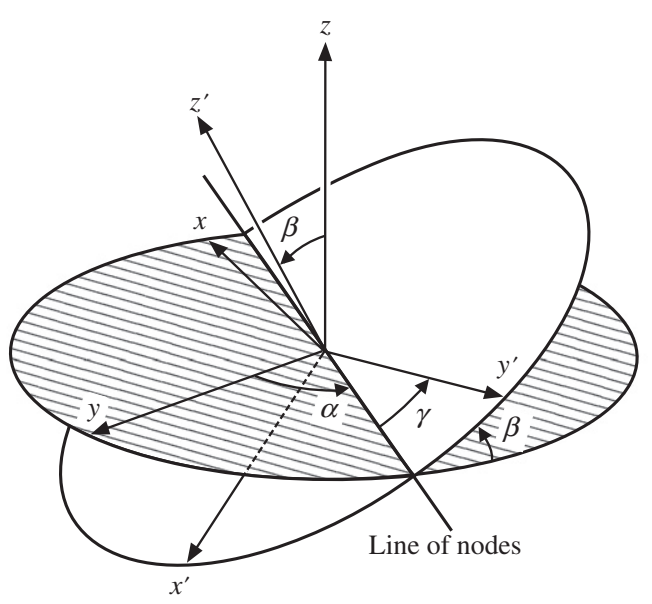

Fig. 5. Euler angles of rotation transforming the laboratory coordinate system $\{x, y, z\}$ into the particle coordinate system $\left\{x^{\prime}, y^{\prime}, z^{\prime}\right\}$. The $z^{\prime}$ axis for the spheroid and the cylinder is directed along the axis of rotational symmetry.

the speckle is the complex exponential factor $\exp \left(-\mathrm{i} k_{1} \hat{\mathbf{n}}^{\mathrm{sca}} \cdot \mathbf{r}^{\prime}\right)$ on the right-hand side of Eq. (10). Indeed, the electric field contributions from two arbitrary elementary volumes of the scattering object centered at $\mathbf{r}^{\prime}$ and $\mathbf{r}^{\prime \prime}$ interfere in the far-field zone, the result of the interference being controlled by the product $\exp \left(-\mathrm{i} k_{1} \hat{\mathbf{n}}^{\mathrm{sca}} \cdot \mathbf{r}^{\prime}\right)\left[\exp \left(-\mathrm{i} k_{1} \hat{\mathbf{n}}^{\mathrm{sca}} \cdot \mathbf{r}^{\prime \prime}\right)\right]^{*}=$ $\exp \left[-\mathrm{i} k_{1} \hat{\mathbf{n}}^{\text {sca }} \cdot\left(\mathbf{r}^{\prime}-\mathbf{r}^{\prime \prime}\right)\right]$, where the asterisk denotes a complex-conjugate value. Depending on the angle between $\hat{\mathbf{n}}^{\text {sca }}$ and $\mathbf{r}^{\prime}-\mathbf{r}^{\prime \prime}$ and on $\left|\mathbf{r}^{\prime}-\mathbf{r}^{\prime \prime}\right|$, this complex exponential can be a rapidly varying function of $\hat{\mathbf{n}}^{\text {sca }}$. As a result, the angular scattering pattern in the farfield zone can be expected to be a superposition of multiple maxima and minima generated by different pairs of elementary volume elements of the scatterer. The most rapidly changing component of the scattering pattern should be caused by the pairs of elementary volume elements with $\left(\mathbf{r}^{\prime}-\mathbf{r}^{\prime \prime}\right) \perp \hat{\mathbf{n}}^{\text {sca }}$ and $\left|\mathbf{r}^{\prime}-\mathbf{r}^{\prime \prime}\right| \approx 2 A_{\min }$, where $A_{\min }$ is the radius of the smallest circumscribing sphere of the scattering object. Therefore, the farfield angular pattern can be expected to vary quite significantly even when the scattering direction changes by as little as $\pi /\left(2 k_{1} A_{\min }\right)$ (rad) since this change corresponds to a change of the phase $k_{1} \hat{\mathbf{n}}^{\text {sca }} \cdot\left(\mathbf{r}^{\prime}-\mathbf{r}^{\prime \prime}\right)$ equal to $\pi$ The actual angular distribution of the intensity maxima and minima depends, of course, on the morphology of the scattering object, as the left-hand panels of Fig. 6 vividly demonstrate. 

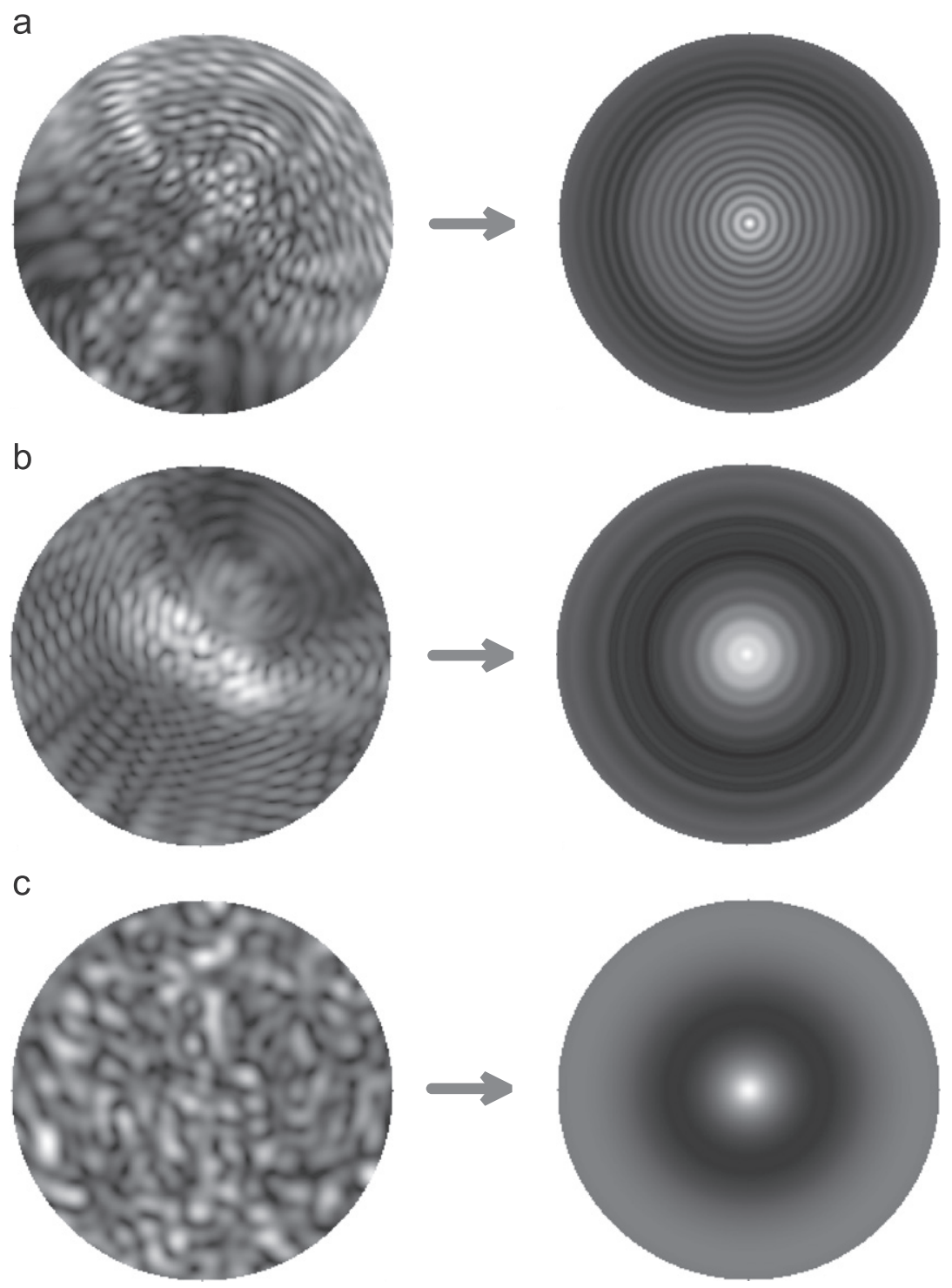

Fig. 6. Total intensity scattered by the spheroid (a), the cylinder (b), and the spherical volume (c) in fixed (left-hand panels) and random (right-hand panels) orientations. The grey scale was individually adjusted in order to maximally reveal the fine structure of each scattering pattern. Fig. 7 shows the angular coordinates used for all six panels.

The speckle patterns in Figs. 6a and $\mathrm{b}$ appear to be somewhat less irregular than that in Fig. 6c. This can be explained qualitatively by the greater morphological complexity of the random multi-sphere group, the less regular spatial distribution of the elementary volume elements contributing to the integral on the right-hand side of Eq. (10), and the fact that empty spaces between the constituent spheres do not contribute to the scattered field.

The right-hand panels of Fig. 6 show the results of averaging the scattered intensity over the uniform orientation distribution of the respective objects. Not surprisingly, the average intensity distributions are rotationally symmetric relative to the incidence direction.

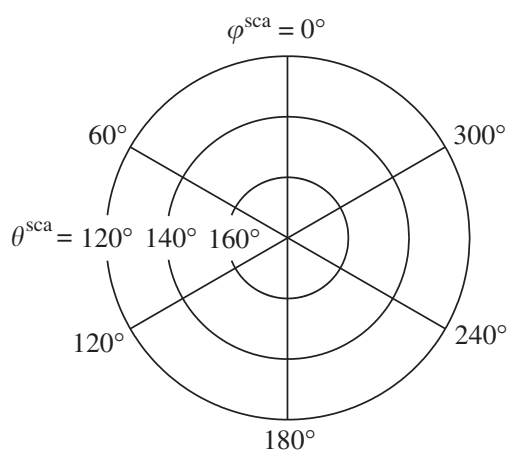

Fig. 7. Angular coordinates used in Fig. 6. 
The scattering patterns for the randomly oriented spheroid and cylinder show residual interference effects, whereas that for the randomly oriented multi-particle group is rather featureless, with the exception of a strong and narrow backscattering peak. Note that the dotted curve in Fig. 3 largely reproduces the radial profile of the scattered intensity in the right-hand panel of Fig. $6 \mathrm{~b}$, the small differences resulting from the different definitions of the particle size parameter (in terms of those of the surface- and volume-equivalent sphere, respectively).

Fig. 6 demonstrates that there can be vast differences between instantaneous or statistically averaged scattering patterns generated by different objects. However, these differences result from differences in the objects' characteristics (such as the overall size relative to the wavelength, morphology, and relative refractive index) rather than from differences in fundamental physical laws describing electromagnetic scattering. The physics of electromagnetic scattering, as embodied by the MMEs or the mathematically equivalent VIE, remains the same irrespective of the nature of the scattering object. To the extent that analytical or numerically-exact solutions of these primordial equations are possible in practice, they incorporate and represent all the physics of the scattering problem.

Despite this irrefutable fact, there is a tendency to believe that the "multi-particle" morphology shown in Fig. 4c somehow results in a multiple-scattering object, whereas the "single-particle" morphologies shown in Figs. 4a and b result in single-scattering objects. We have mentioned, however, that multiple electromagnetic scattering is not a real physical phenomenon. Indeed, all three left-hand panels in Fig. 6 are the result of solving the same MMEs and describe single electromagnetic scattering by three morphologically different objects. The computation of the left-hand panel in Fig. 6c did not involve any new equations and, thus, any new physical phenomena.

However, multiple scattering can be viewed as a useful mathematical abstraction facilitating, in particular, the derivation of such important honest theories as the microphysical theories of radiative transfer (RT) and coherent backscattering (CB). The specific way to introduce the mathematical concept of multiple scattering is as follows.

Consider an arbitrary scattering object, e.g., the spheroid shown in Fig. 4a, and subdivide it arbitrarily into a number $N$ of non-overlapping volume elements $V_{i}$ such that

$V_{\mathrm{INT}}=\bigcup_{i=1}^{N} V_{i}$

The volume elements need not be infinitesimally small. The next step is to represent the total scattered field at an observation point $\mathbf{r}$ as a vector superposition of partial fields scattered by the individual volume elements:

$\mathbf{E}(\mathbf{r})=\mathbf{E}^{\mathrm{inc}}(\mathbf{r})+\sum_{i=1}^{N} \mathbf{E}_{i}^{\mathrm{sca}}(\mathbf{r}), \quad \mathbf{r} \in \mathfrak{R}^{3}$,

where $\mathbf{E}_{i}^{\text {sca }}(\mathbf{r})$ is the $i$ th partial scattered electric field. Then it can be shown that the partial scattered fields are found by solving vector so-called Foldy-Lax equations (FLEs) which follow directly from the VIE and are exact $[7,50]$. Specifically, the $i$ th partial scattered field is given by

$\mathbf{E}_{i}^{\mathrm{sca}}(\mathbf{r})=\int_{V_{i}} \mathrm{~d} \mathbf{r}^{\prime} \stackrel{\leftrightarrow}{G}\left(\mathbf{r}, \mathbf{r}^{\prime}\right) \cdot \int_{V_{i}} \mathrm{~d} \mathbf{r}^{\prime \prime} \stackrel{\leftrightarrow}{T}{ }_{i}\left(\mathbf{r}^{\prime}, \mathbf{r}^{\prime \prime}\right) \cdot \mathbf{E}_{i}\left(\mathbf{r}^{\prime \prime}\right)$,

where $\mathbf{E}_{i}\left(\mathbf{r}_{\leftrightarrow}^{\prime \prime}\right)$ is the electric field "exciting" volume $i$, and the $N$ dyadics $T_{i}$ are found by solving individually the following Lippmann-Schwinger equation:

$$
\begin{aligned}
\stackrel{\leftrightarrow}{T}_{i}\left(\mathbf{r}, \mathbf{r}^{\prime}\right)= & k_{1}^{2}\left[m_{i}^{2}(\mathbf{r})-1\right] \delta\left(\mathbf{r}-\mathbf{r}^{\prime}\right) \stackrel{\leftrightarrow}{I} \\
& +k_{1}^{2}\left[m_{i}^{2}(\mathbf{r})-1\right] \int_{V_{i}} \mathrm{~d} \mathbf{r}^{\prime \prime} \stackrel{\leftrightarrow}{G}\left(\mathbf{r}, \mathbf{r}^{\prime \prime}\right) \cdot \stackrel{\leftrightarrow}{T}_{i}\left(\mathbf{r}^{\prime \prime}, \mathbf{r}^{\prime}\right), \quad \mathbf{r}, \mathbf{r}^{\prime} \in V_{i},
\end{aligned}
$$

where $\delta(\mathbf{r})$ is the three-dimensional delta function. The $\stackrel{\leftrightarrow}{T}_{i}$ is the dyadic transition operator of the $i$ th volume element with respect to the fixed laboratory coordinate system computed in the absence of all the other volume elements. Thus, the $N$ dyadic transition operators are totally independent of each other. However, the exciting fields are interdependent and are found by solving the following system of $N$ linear integral equations:

$\mathbf{E}_{i}(\mathbf{r})=\mathbf{E}^{\mathrm{inc}}(\mathbf{r})+\sum_{j(\neq i)=1}^{N} \mathbf{E}_{i j}(\mathbf{r}), \quad \mathbf{r} \in V_{i}, i=1, \ldots, N$,

where the "partial" exciting fields $\mathbf{E}_{i j}(\mathbf{r})$ are given by

$\mathbf{E}_{i j}(\mathbf{r})=\int_{V_{j}} \mathrm{~d} \mathbf{r}^{\prime} \stackrel{\leftrightarrow}{G}\left(\mathbf{r}, \mathbf{r}^{\prime}\right) \cdot \int_{V_{j}} \mathrm{~d} \mathbf{r}^{\prime \prime} \stackrel{\leftrightarrow}{T} j\left(\mathbf{r}^{\prime}, \mathbf{r}^{\prime \prime}\right) \cdot \mathbf{E}_{j}\left(\mathbf{r}^{\prime \prime}\right)$

The FLEs (12)-(16) are equivalent to the VIE. However, the fact that $\stackrel{\leftrightarrow}{T}_{i}$ for each $i$ is an individual property of the $i$ th volume element computed as if this volume element were alone allows one to introduce the mathematical concept of multiple scattering. Indeed, let us rewrite Eqs. (12), (13), (15), and (16) in a compact operator form:

$E=E^{\text {inc }}+\sum_{i=1}^{N} \hat{G} \hat{T}_{i} E_{i}$,

$E_{i}=E^{\mathrm{inc}}+\sum_{j(\neq i)=1}^{N} \hat{G} \hat{T}_{j} E_{j}$

where

$\hat{G} \hat{T}_{j} E_{j}=\int_{V_{j}} \mathrm{~d} \mathbf{r}^{\prime} \stackrel{\leftrightarrow}{G}\left(\mathbf{r}, \mathbf{r}^{\prime}\right) \cdot \int_{V_{j}} \mathrm{~d} \mathbf{r}^{\prime \prime} \stackrel{\leftrightarrow}{T}{ }_{j}\left(\mathbf{r}^{\prime}, \mathbf{r}^{\prime \prime}\right) \cdot \mathbf{E}_{j}\left(\mathbf{r}^{\prime \prime}\right)$

Iterating Eq. (18) yields

$$
\begin{aligned}
E_{i}= & E^{\text {inc }}+\sum_{j(\neq i)=1}^{N} \hat{G} \hat{T}_{j} E^{\text {inc }}+\sum_{\substack{j \neq i)=1 \\
l \neq j)=1}}^{N} \hat{G} \hat{T}_{j} \hat{G} \hat{T}_{l} E^{\text {inc }} \\
& +\sum_{\substack{j(\neq i)=1 \\
l \neq j)=1 \\
m(\neq l)=1}}^{N} \hat{G} \hat{T}_{j} \hat{G} \hat{T}_{l} \hat{G} \hat{T}_{m} E^{\text {inc }}+\cdots,
\end{aligned}
$$

whereas the substitution of Eq. (20) in (17) gives

$E=E^{\mathrm{inc}}+E^{\mathrm{sca}}$, 


$$
\begin{gathered}
E^{\text {sca }}=\sum_{i=1}^{N} \hat{G} \hat{T}_{i} E^{\text {inc }}+\sum_{\substack{i=1 \\
j(\neq i)=1}}^{N} \hat{G} \hat{T}_{i} \hat{G} \hat{T}_{j} E^{\text {inc }} \\
+\sum_{\substack{i=1 \\
j(\neq i)=1 \\
l \neq j)=1}}^{N} \hat{G} \hat{T}_{i} \hat{G} \hat{T}_{j} \hat{G} \hat{T}_{l} E^{\mathrm{inc}}+\cdots
\end{gathered}
$$

The last two equations are nothing more than what is usually called the Newmann or the Born expansion of the total field. However, if one really wishes to introduce the concept of multiple scattering, it is these formulas that can be interpreted, purely mathematically, as an order-of-scattering expansion. Indeed, the dyadic transition operators are independent of each other, and each of them can be characterized as a unique and complete electromagnetic identifier of the corresponding volume element. Therefore, $\hat{G} \hat{T}_{i} E^{\text {inc }}$ can be interpreted as the partial scattered filed at the observation point generated by the $i$ th volume element in response to the excitation by the incident field only, $\hat{G} \hat{T}_{i} \hat{G} \hat{T}_{j} E^{\text {inc }}$ is the partial field generated by the same volume element in response to the excitation caused by the $j$ th volume element in response to the excitation by the incident field, etc. Thus, the first term on the right-hand side of Eq. (22) can be interpreted as the sum of all single-scattering contributions, the second term is the sum of all doublescattering contributions, etc. The first term on the righthand side of Eq. (21) represents the unscattered (i.e., the incident) field.

We intentionally began the discussion of the FLEs by referring to a scattering object in the form of a spheroid. Indeed, no one would claim that a spheroid is a multiplescattering object, which demonstrates once again that the FLEs and their order-of-scattering representation do not describe multiple scattering as a real physical phenomenon. For example, the term $\hat{G} \hat{T}_{i} \hat{G} \hat{T}_{j} \hat{G} \hat{T}_{l} E^{\text {inc }}$ on the right-hand side of Eq. (22) cannot be interpreted by saying that "the incident wave approaches volume element $l$, gets scattered by volume element $l$ towards volume element $j$, approaches volume element $j$, gets scattered by volume element $j$ towards volume element $i$, approaches volume element $i$, gets scattered by volume element $i$ towards the observation point, and finally arrives at the observation point". Indeed, it follows from Eqs. (15) and (16) that all mutual excitations $\mathbf{E}_{i j}(\mathbf{r})$ occur simultaneously and are not temporally discrete and ordered events. Furthermore, they are not, in general, electromagnetic waves and do not propagate in a specific direction. All this shows again that Eqs. (21) and (22) are nothing more than a mathematical expansion of the total field in a certain series.

In the limit $N \rightarrow \infty, V_{i} \rightarrow 0 \forall i$ the FLEs yield the same result as a widely used numerically-exact method called the discrete dipole approximation [26,51]. Otherwise they may appear to be too complicated to find extensive practical applications. It turns out, however, that the order-of-scattering form of the FLEs is a convenient mathematical tool that can be used to derive the honest microphysical theories of RT and CB. This recent development will be discussed in the following section.

\section{Microphysical theories of radiative transfer and coherent backscattering}

The phenomenological RTT pioneered by Lommel, Chwolson, and Schuster [52-54] is a thoroughly phenomenological theory based on an eclectic combination of principles and concepts borrowed on an ad hoc basis from honest theories such as classical electromagnetics and QED as well as from phenomenological approaches such as the GOM and classical radiometry. The numerous inherent inconsistencies and the overall inadequacy of the phenomenological RTT have been exposed and extensively discussed in $[7,8,25,55]$. Importantly, however, the phenomenological RTT, as applied to a DRM composed of randomly positioned macroscopic particles, has been replaced by the honest microphysical RTT [56,57] and, thus, has been rendered unnecessary.

The natural way to start the derivation of the microphysical theories of RT and CB is to apply the FLEs to an $N$ particle group (Fig. 1) and choose $N$ volumes $V_{i}$ such that each of them coincides with the interior of an entire particle. As a consequence, the $\overleftrightarrow{T}_{i}$ in Eq. (13) becomes the dyadic transition operator of the entire $i$ th particle with respect to the fixed laboratory coordinate system computed as if all the other particles did not exist. In other words, the $T_{i}$ now serves as a complete and unique electromagnetic identifier of particle $i$.

The resulting FLEs and their order-of-scattering form can be greatly simplified by making the following two assumptions:

- The $N$ particles forming the group are separated widely enough that each of them is located in the far-field zones of all the other particles.

- The observation point is located in the far-field zone of any particle from the group.

Indeed, the partial contribution of the $j$ th particle to the field exciting the $i$ th particle in Eq. (15) now reduces to a simple outgoing spherical wavelet centered at the origin of particle $j$. The radius of curvature of this wavelet at the origin of particle $i$ is much larger than the size of particle $i$ so that the wavelet can be effectively considered as locally plane and locally homogeneous. Hence the scattering of this wavelet by particle $i$ can be described in terms of the conventional far-field scattering dyadic $A_{i}$ centered at the origin of particle $i[7,36]$. As a consequence, the original system of the integral FLEs is converted into a system of algebraic equations, while the original order-of-scattering expansion becomes purely algebraic.

Specifically, assuming that the incident field is a plane electromagnetic wave propagating in the direction of the unit vector $\hat{\mathbf{n}}^{\text {inc }}$, we have for the total field at an observation point $\mathbf{r}$ located in the respective far-field zones of all the $N$ particles [7]:

$$
\begin{aligned}
\mathbf{E}(\mathbf{r})= & \mathbf{E}^{\mathrm{inc}}(\mathbf{r})+\sum_{i=1}^{N} G\left(r_{i}\right) \overleftrightarrow{A}_{i}\left(\hat{\mathbf{r}}_{i}, \hat{\mathbf{n}}^{\mathrm{inc}}\right) \cdot \mathbf{E}^{\mathrm{inc}}\left(\mathbf{R}_{i}\right) \\
& +\sum_{i=1}^{N} G\left(r_{i}\right) \sum_{j(\neq i)=1}^{N} \overleftrightarrow{A}_{i}\left(\hat{\mathbf{r}}_{i}, \hat{\mathbf{R}}_{i j}\right) \cdot \mathbf{E}_{i j},
\end{aligned}
$$


where $G(r)=\exp \left(\mathrm{i} k_{1} r\right) / r, r_{i}$ is the distance between the origin of particle $i$ and the observation point, $\hat{\mathbf{r}}_{i}$ is the unit vector directed from the origin of particle $i$ towards the observation point, $\mathbf{R}_{i}$ is the position vector of the $i$ th particle origin, $\hat{\mathbf{R}}_{i j}$ is the unit vector directed from the origin of particle $j$ towards the origin of particle $i$ (Fig. 8), and $\mathbf{E}_{i j}$ are the distance-independent amplitudes of the mutual particle-particle excitations. Eq. (23) shows that the total field at any observation point located sufficiently far from any particle in the group is the superposition of the incident plane wave and $N$ spherical waves generated by and centered at the $N$ particles. The $\mathbf{E}_{i j}$ are now found by solving a system of $N(N-1)$ linear algebraic (rather than integral!) equations [7]:

$$
\begin{aligned}
\mathbf{E}_{i j}= & G\left(R_{i j}\right) \overleftrightarrow{A}_{j}\left(\hat{\mathbf{R}}_{i j}, \hat{\mathbf{n}}^{\mathrm{inc}}\right) \cdot \mathbf{E}^{\mathrm{inc}}\left(\mathbf{R}_{j}\right) \\
& +G\left(R_{i j}\right) \sum_{l(\neq j)=1}^{N} \stackrel{\leftrightarrow}{A}_{j}\left(\hat{\mathbf{R}}_{i j}, \hat{\mathbf{R}}_{j l}\right) \cdot \mathbf{E}_{j l}, \quad i, j=1, \ldots, N, j \neq i,
\end{aligned}
$$

where $R_{i j}$ is the distance between the origins of particles $j$ and $i$. The resulting order-of-scattering expansion of the total field also becomes much simpler:

$$
\begin{aligned}
\mathbf{E}= & \mathbf{E}^{\mathrm{inc}}+\sum_{i=1}^{N} \stackrel{\leftrightarrow}{B}_{r i 0} \cdot \mathbf{E}_{i}^{\mathrm{inc}}+\sum_{i=1}^{N} \sum_{j(\neq i)=1}^{N} \stackrel{\leftrightarrow}{B}_{r i j} \cdot \stackrel{\leftrightarrow}{B}_{i j 0} \cdot \mathbf{E}_{j}^{\mathrm{inc}} \\
& +\sum_{i=1}^{N} \sum_{j(\neq i)=1}^{N} \sum_{l(\neq j)=1}^{N} \stackrel{\leftrightarrow}{B}_{r i j} \cdot \stackrel{\leftrightarrow}{B}_{i j l} \cdot \stackrel{\leftrightarrow}{B}_{j l 0} \cdot \mathbf{E}_{l}^{\mathrm{inc}}+\cdots,
\end{aligned}
$$

where

$$
\begin{aligned}
& \mathbf{E}=\mathbf{E}(\mathbf{r}), \quad \mathbf{E}^{\mathrm{inc}}=\mathbf{E}^{\mathrm{inc}}(\mathbf{r}), \quad \mathbf{E}_{i}^{\mathrm{inc}}=\mathbf{E}^{\mathrm{inc}}\left(\mathbf{R}_{i}\right), \\
& \overleftrightarrow{B}_{r i 0}=G\left(r_{i}\right) \stackrel{\leftrightarrow}{A}_{i}\left(\hat{\mathbf{r}}_{i}, \hat{\mathbf{n}}^{\mathrm{inc}}\right), \\
& \overleftrightarrow{B}_{r i j}=G\left(r_{i}\right) \stackrel{\leftrightarrow}{A}_{i}\left(\hat{\mathbf{r}}_{i}, \hat{\mathbf{R}}_{i j}\right), \\
& \overleftrightarrow{B}_{i j 0}=G\left(R_{i j}\right) \stackrel{\leftrightarrow}{A}_{j}\left(\hat{\mathbf{R}}_{i j}, \hat{\mathbf{n}}^{\mathrm{inc}}\right), \\
& \stackrel{\leftrightarrow}{B}_{i j l}=G\left(R_{i j}\right) \stackrel{\leftrightarrow}{A}_{j}\left(\hat{\mathbf{R}}_{i j}, \hat{\mathbf{R}}_{j l}\right) .
\end{aligned}
$$

One can see indeed that now the role of the unique electromagnetic identifier of each particle is assumed by the corresponding particle-centered scattering dyadic, that is, the same quantity that would completely describe far-field scattering of a plane electromagnetic wave by this particle if it were solitary rather than a member of the group. In other words, the far-field scattering dyadics $A_{i}(i=1, \ldots, N)$ are computed by solving the MMEs separately for each particle, which is much simpler than solving the MMEs for the entire multiparticle group.

Each term on the right-hand side of Eq. (25) can be viewed as a multi-particle wave path resulting in a partial transverse electromagnetic wave at the observation point. Each partial wave is characterized by a well-defined phase obtained by evaluating the respective product of the participating $G$-factors. This means that one can evaluate the result of the interference of any pair of such "multiply-scattered" waves at the observation point as a function of the phase difference between the waves.

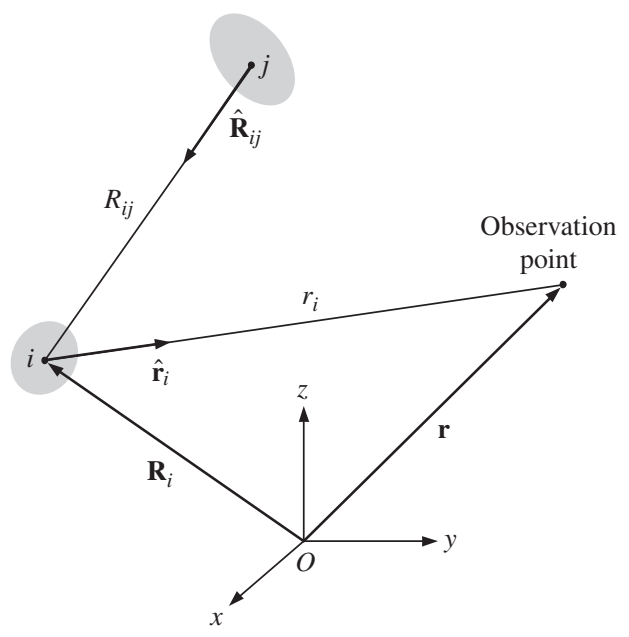

Fig. 8. Scattering contribution caused by particle $i$ in response to excitation by particle $j$.

Most typically, random movements of the constituent particles during the measurement cause a randomly varying phase difference between two multi-particle wave paths such as those shown in Fig. 4g. As a consequence, averaging over particle positions yields a zero net result.

There are, however, two classes of wave pairs whose contributions survive the averaging over particle coordinates. Fig. $4 \mathrm{~h}$ gives an example of the so-called ladder diagram [58] wherein both multi-particle wave paths are self-avoiding (i.e., are not allowed to go through a particle more than once) and go through the same string of particles. In this case the corresponding phase difference is identically equal to zero irrespective of particle positions, thereby causing constantly constructive interference. The summation of all ladder-diagram contributions in the limit $N \rightarrow \infty$ coupled with averaging over the uniform distribution of particle positions throughout the scattering medium yields the vector radiative transfer equation (RTE) [56,57]. The latter is valid at any observation point except far-field observation points located in the exact forward-scattering direction.

Another class of wave pairs causing a non-zero contribution in the far-field zone of the entire scattering object is exemplified in Fig. 4i. Such pairs of self-avoiding wave paths are called cyclical diagrams [58]. In this case the two conjugate wave paths go through the same string of particles, but in opposite directions. The corresponding phase difference at a remote observation point is given by

$\Delta=k_{1}\left(\mathbf{R}_{n}-\mathbf{R}_{1}\right) \cdot\left(\hat{\mathbf{n}}^{\mathrm{inc}}+\hat{\mathbf{n}}^{\mathrm{sca}}\right)$.

If the observation direction $\hat{\mathbf{n}}^{\text {sca }}$ is far from the exact backscattering direction given by $-\hat{\mathbf{n}}^{\text {inc }}$ then $\Delta$ varies randomly with particle coordinates, and so the average effect of the interference is zero. However, at exactly the backscattering direction, $\hat{\mathbf{n}}^{\text {sca }}=-\hat{\mathbf{n}}^{\text {inc }}$, the phase difference between the conjugate wave paths involving any string of particles is identically equal to zero, and the 
interference is always constructive, thereby causing an intensity peak. The summation of all cyclical-diagram contributions in the limit $N \rightarrow \infty$ coupled with averaging over the uniform distribution of particle positions throughout the scattering medium yields the microphysical theory of CB [7].

The classical integro-differential form of the RTE reads

$$
\begin{aligned}
\hat{\mathbf{q}} \cdot \nabla \tilde{\mathbf{I}}(\mathbf{r}, \hat{\mathbf{q}})= & -n_{0}\langle\mathbf{K}(\hat{\mathbf{q}})\rangle_{\xi} \tilde{\mathbf{I}}(\mathbf{r}, \hat{\mathbf{q}}) \\
& +n_{0} \int_{4 \pi} \mathrm{d} \hat{\mathbf{q}}^{\prime}\left\langle\mathbf{Z}\left(\hat{\mathbf{q}}, \hat{\mathbf{q}}^{\prime}\right)\right\rangle_{\xi} \tilde{\mathbf{I}}\left(\mathbf{r}, \hat{\mathbf{q}}^{\prime}\right) .
\end{aligned}
$$

In this equation, $\tilde{\mathbf{I}}(\mathbf{r}, \hat{\mathbf{q}})$ is the $4 \times 4$ so-called specific intensity column vector, $\langle\mathbf{K}(\hat{\mathbf{q}})\rangle_{\xi}$ and $\left\langle\mathbf{Z}\left(\hat{\mathbf{q}}, \hat{\mathbf{q}}^{\prime}\right)\right\rangle_{\xi}$ are the extinction and the phase matrix per particle, respectively, averaged over all particle states (i.e., sizes, shapes, orientations, and relative refractive indices), and $n_{0}=N / V$ is the particle number density. The specific intensity column vector is a function of spatial coordinates of the observation point $\mathbf{r}$ and the "propagation direction" $\hat{\mathbf{q}}$ and can be decomposed into the coherent and diffuse parts,

$\tilde{\mathbf{I}}(\mathbf{r}, \hat{\mathbf{q}})=\delta\left(\hat{\mathbf{q}}-\hat{\mathbf{n}}^{\mathrm{inc}}\right) \mathbf{I}_{\mathrm{c}}(\mathbf{r})+\tilde{\mathbf{I}}_{\mathrm{d}}(\mathbf{r}, \hat{\mathbf{q}})$,

each satisfying its own RTE:

$$
\begin{aligned}
& \hat{\mathbf{n}}^{\text {inc }} \cdot \nabla \mathbf{I}_{\mathrm{c}}(\mathbf{r})=-n_{0}\left\langle\mathbf{K}\left(\hat{\mathbf{n}}^{\text {inc }}\right)\right\rangle_{\xi} \mathbf{I}_{\mathrm{c}}(\mathbf{r}), \\
& \hat{\mathbf{q}} \cdot \nabla \tilde{\mathbf{I}}_{\mathrm{d}}(\mathbf{r}, \hat{\mathbf{q}})=-n_{0}\langle\mathbf{K}(\hat{\mathbf{q}})\rangle_{\xi} \tilde{\mathbf{I}}_{\mathrm{d}}(\mathbf{r}, \hat{\mathbf{q}}) \\
& \quad+n_{0} \int_{4 \pi} \mathrm{d} \hat{\mathbf{q}}^{\prime}\left\langle\mathbf{Z}\left(\hat{\mathbf{q}}, \hat{\mathbf{q}}^{\prime}\right)\right\rangle_{\xi} \tilde{\mathbf{I}}_{\mathrm{d}}\left(\mathbf{r}, \hat{\mathbf{q}}^{\prime}\right)+n_{0}\left\langle\mathbf{Z}\left(\hat{\mathbf{q}}, \hat{\mathbf{n}}^{\mathrm{inc}}\right)\right\rangle_{\xi} \mathbf{I}_{\mathrm{c}}(\mathbf{r}),
\end{aligned}
$$

where $\delta(\hat{\mathbf{q}})$ is the solid-angle delta function. The coherent column vector $\mathbf{I}_{\mathrm{c}}$ reduces to the Stokes column vector of the incident plane wave at the illuminated boundary of the medium, but is subject to exponential attenuation and, possibly, the effect of dichroism inside the medium.

According to Eq. (33), the fundamental difference between the coherent Stokes column vector $\mathbf{I}_{c}$ and the diffuse specific intensity column vector $\tilde{\mathbf{I}}_{\mathrm{d}}$ is that the former describes a monodirectional whereas the latter describes an uncollimated flow of electromagnetic energy. For example, the first element of the coherent Stokes column vector, i.e., the coherent intensity $I_{\mathrm{c}}(\mathbf{r})$ is the electromagnetic power per unit area of a small surface element $\Delta S$ perpendicular to the incidence direction $\hat{\mathbf{n}}^{\text {inc }}$, whereas the first element of the diffuse specific intensity column vector, i.e., the diffuse specific intensity $\tilde{I}_{\mathrm{d}}(\mathbf{r}, \hat{\mathbf{q}})$, is the electromagnetic power per unit area of a small surface element $\Delta S$ perpendicular to $\hat{\mathbf{q}}$ per one steradian of a small solid angle $\Delta \Omega$ centered around $\hat{\mathbf{q}}$. This interpretation of $\mathbf{I}_{\mathrm{c}}(\mathbf{r})$ and $\tilde{\mathbf{I}}_{\mathrm{d}}(\mathbf{r}, \hat{\mathbf{q}})$ implies that both quantities can be measured by appropriately placed and oriented detectors of electromagnetic energy flux, which explains the practical usefulness of the RTT.

\section{Range of applicability of the honest theories of radiative transfer and coherent backscattering}

The discussion in the preceding section demonstrates that RT and CB are idealized mathematical concepts valid under specific circumstances. Since the microphysical theories of RT and CB follow directly from the MMEs upon making a sequence of well-defined assumptions $[7,8]$, their practical applicability does not require any validation provided that all these assumptions are fulfilled. For example, there is little if any doubt that the RTE describes adequately the results of photopolarimetric observations of clouds in planetary atmospheres, as exemplified by the remote-sensing discovery of micrometer-sized sulfuric acid droplets in the atmosphere of Venus [59]. However, if either theory is used to model situations in which one or more of the underlying assumptions are violated then its quantitative applicability must be carefully and extensively examined. The requisite test results can be the outcome of a direct numerically-exact solution of the MMEs or a controlled laboratory experiment.

For example, one of the basic assumptions that one has to make in order to arrive at the mathematical concepts of RT and CB is that the particles forming a DRM are located in the far-field zones of each other, which implies a very low particle packing density. However, both concepts have been used frequently to describe electromagnetic scattering by densely packed DRMs such as particle suspensions and particulate surfaces with volume packing densities comparable to or even exceeding $10 \%$.

One way to validate the quantitative applicability of the concepts of RT and CB to such densely packed DRMs is to compare the results of numerical computations with those of controlled laboratory experiments. As discussed in [3], there is a fundamental difference between controlled laboratory measurements and laboratory observations. In the former, one fully controls all the conditions of the experiment and has complete independent knowledge of all physical parameters specifying the scattering medium (e.g., the size distribution, shape, refractive index, and packing density of the particles and the geometrical dimensions of the scattering medium) as well as the capability to change them one at a time. The known parameters of the medium then serve as input for numerical computations of electromagnetic scattering, thereby making possible a direct and unambiguous comparison of theoretical and measurement results. Laboratory observations do not differ from remote-sensing (e.g., astronomical) observations in that one measures only the parameters of the scattered light, without providing independently a complete physical and compositional specification of the scattering medium. As such, laboratory observations cannot be used for validation purposes. Instructive examples of the use of controlled laboratory measurements of electromagnetic scattering for validating the applicability of the low-packing-density concepts of RT and CB to densely packed DRMs can be found, e.g., in [60-62].

Another way to test the applicability of an honest lowdensity theory of electromagnetic scattering to a densely packed DRM is to use the results of direct computer solutions of the MMEs. There are three numerically-exact computer solvers of the MMEs that have been applied recently to model electromagnetic scattering by media consisting of large numbers of randomly positioned particles: the superposition $T$-matrix method $[36,49]$, 

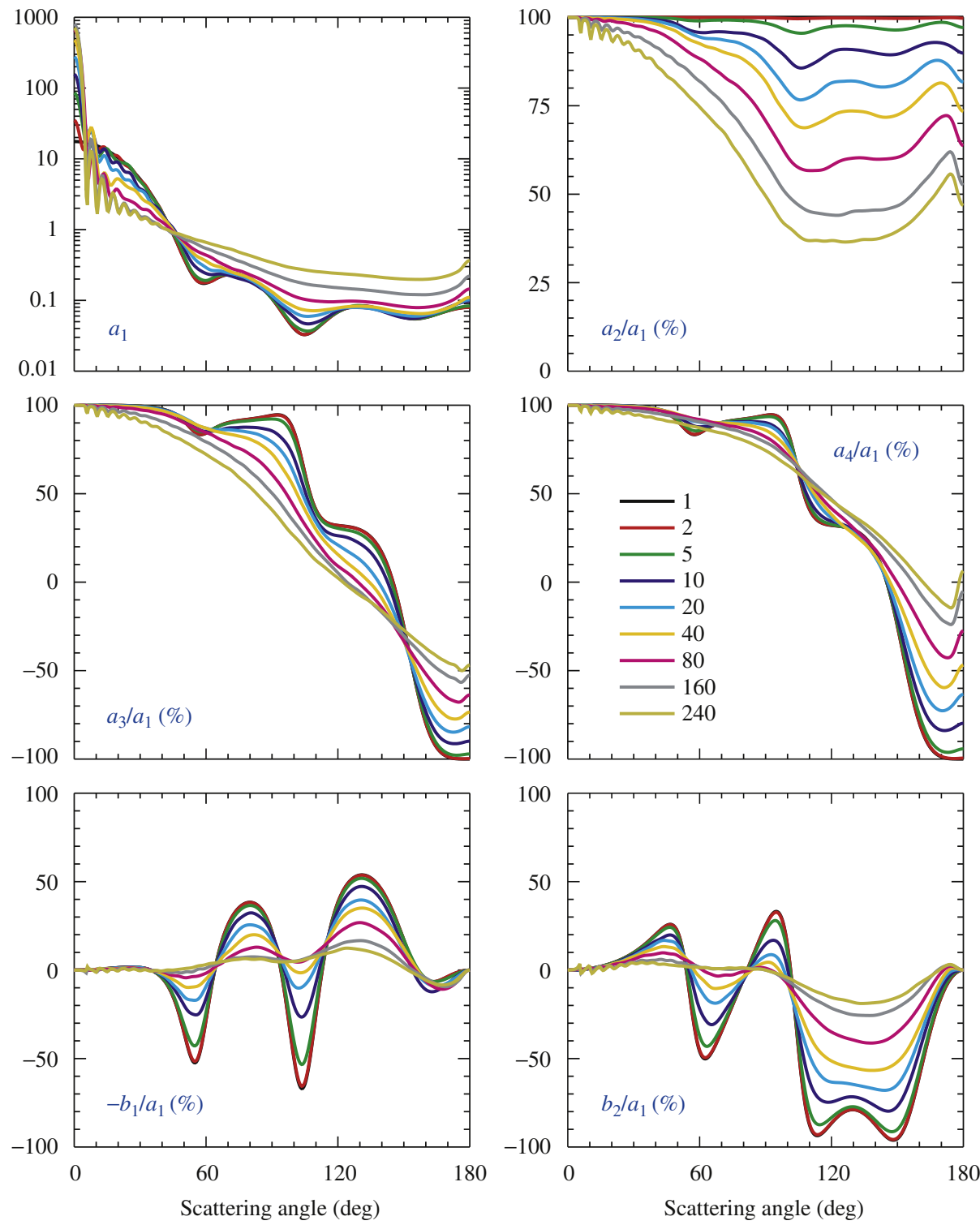

Fig. 9. Elements of the normalized Stokes scattering matrix computed for a $k_{1} A=40$ spherical volume of discrete random medium filled with $N=1, \ldots, 240$ identical spherical particles. The relative refractive index and size parameter of the particles are fixed at $k_{1} a=4$ and $m=1.32$.

the finite-difference time-domain method [63,64], and the discrete dipole approximation [26,51]. By directly solving the MMEs one can

- generate benchmark numbers by eliminating any uncertainty associated with the use of an approximate honest theoretical approach or a phenomenological theory;

- control precisely all physical parameters of the scattering medium and vary them one at a time;

- compute all requisite optical observables at once.

Such modeling can be viewed as an ideal controlled laboratory experiment in which one studies unambiguously the onset, evolution, and potential decay of all observable manifestations of RT and $\mathrm{CB}$ as the particle packing density gradually increases from zero to values typical of actual particulate surfaces and particle suspensions. Numerous applications of this approach can be found in [65-79].

The right-hand panel of Fig. 6c gives an example of how a direct numerically-exact solution of the MMEs reproduces, at least qualitatively, the main prediction of the low-density theory of $\mathrm{CB}$, viz., the strong and narrow backscattering intensity peak. Indeed, by averaging the scattered intensity over all orientations of the multiparticle group shown in Fig. 4c, one effectively models a macroscopic scattering volume filled with randomly positioned particles.

Another example of numerically-exact $T$-matrix results is given in Fig. 9, which depicts the far-field angular 


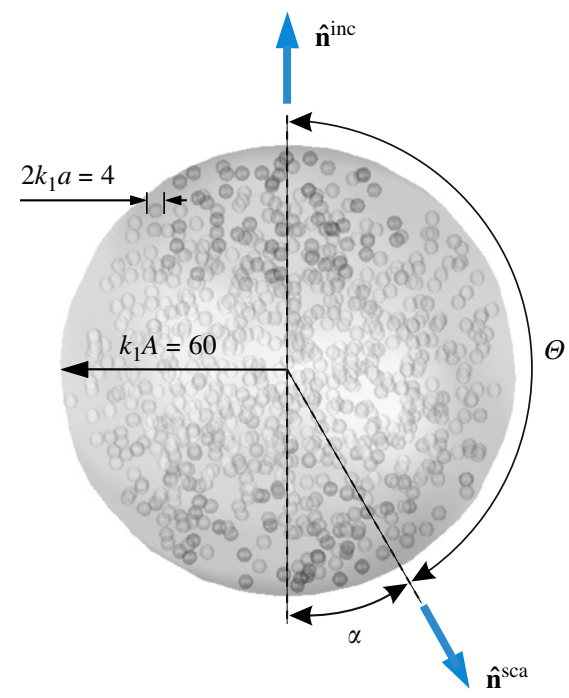

Fig. 10. Far-field scattering by a macroscopic spherical volume of DRM. The normalized Stokes scattering matrix is defined with respect to the scattering plane (i.e., the plane through the incidence and scattering directions) as a function of the scattering angle $\Theta$. In this case the $k_{1} A=60$ spherical volume is populated by $N=800$ identical spherical particles having a size parameter $k_{1} a=2$.

dependence of the elements of the normalized Stokes scattering matrix $\tilde{\mathbf{F}}(\Theta)$ for a macroscopic spherical volume of DRM populated with increasing numbers of randomly positioned particles [68]. To define the scattering matrix, we make a simplifying assumption that $\varphi^{\text {sca }}=0$ and specify the scattering direction in terms of the scattering angle $\Theta=\theta^{\text {sca }}$ (cf. Figs. 4d and 10). Then, by definition $[36,80]$,

$\tilde{\mathbf{F}}(\Theta)=\left[\begin{array}{cccc}a_{1}(\Theta) & b_{1}(\Theta) & 0 & 0 \\ b_{1}(\Theta) & a_{2}(\Theta) & 0 & 0 \\ 0 & 0 & a_{3}(\Theta) & b_{2}(\Theta) \\ 0 & 0 & -b_{2}(\Theta) & a_{4}(\Theta)\end{array}\right]=\frac{4 \pi}{C_{\text {sca }}} \mathbf{Z}\left(\hat{\mathbf{n}}^{\text {sca }}, \hat{\mathbf{n}}^{\text {inc }}\right)$,

where $C_{\mathrm{sca}}$ is the scattering cross section of the entire volume, and the phase function $a_{1}(\Theta)$ satisfies the normalization condition (8).

As we have already mentioned, the phase function describes the angular distribution of the scattered intensity provided that the incident light is unpolarized. The upper left-hand panel of Fig. 9 vividly demonstrates two fundamental consequences of increasing $N$ consistent, at least qualitatively, with predictions of the lowdensity theories of RT and CB [7,48]. First of all, increasing $N$ makes the phase function at scattering angles $30^{\circ} \leq \Theta \leq 170^{\circ}$ progressively smooth and featureless, thereby causing the "diffuse" intensity background clearly identifiable in the right-hand panel of Fig. 6c. The smoothness of the background intensity can be interpreted as a typical RT consequence of increasing amount of multiple scattering with increasing $N$ whereby light undergoing many "scattering events" forgets the initial incidence direction $\hat{\mathbf{n}}^{\text {inc }}$ and is likely to contribute equally to all "exit" directions $\hat{\mathbf{n}}^{\text {sca }}$. Second of all, the phase functions at scattering angles $\Theta>170^{\circ}$ start to develop a backscattering enhancement which becomes quite pronounced for $N \geq 160$. This feature can be interpreted as a typical manifestation of $\mathrm{CB}$.

The upper left panel of Fig. 9 also reveals a far-field optical effect which the RTT does not reproduce. Specifically, the constructive interference of light singly scattered by the component particles in the exact forward direction causes a strong forward-scattering enhancement in the far-field zone of the entire volume [68]. The origin of this feature is explained in Fig. 4f. Indeed, the exact forward-scattering direction is unique in that the phase of the wavelets singly forward-scattered by all the particles in the volume is exactly the same irrespective of the specific particle positions. In the absence of multiple scattering, the constructive interference of these wavelets would lead to an increase of the forwardscattering phase function $a_{1}\left(0^{\circ}\right)$ by a factor of $N$. This increase does occur for $N=2$ and 5 , but then it slows down and eventually saturates at a value close to the LorenzMie prediction for a homogeneous $k_{1} A=40$ spherical scatterer. Interestingly enough, the extinction cross section of the entire volume also saturates at a value close to twice its projected area $\left(C_{\text {ext }} \rightarrow 2 \pi A^{2}\right)$ by the time the constituent $k_{1} a=4$ particles fill completely the volume's projection. This behavior of $a_{1}\left(0^{\circ}\right)$ and $C_{\text {ext }}$ with increasing $N$ can be interpreted qualitatively in terms of constituent particles "shading" each other. Ultimately the far-field forward-scattering behavior of the scattering volume filled with densely packed, randomly positioned particles starts to resemble that of a projection-area-equivalent homogeneous scatterer.

The degree of linear polarization of the scattered light for unpolarized incident light is given by the ratio $-Q^{\text {sca }} / I^{\text {sca }}=-b_{1} / a_{1}$. The bottom left-hand panel of Fig. 9 shows that the most obvious effect of increasing $N$ is to smooth out the oscillations inherent in the polarization curve for a single $k_{1} a=4$ sphere and, on average, to make polarization more neutral. The traditional RT explanation of this behavior is that the main contribution to $Q^{\text {sca }}$ comes from the first order of scattering, whereas light "scattered many times", as in Fig. 4h, becomes largely unpolarized $[7,48,80]$. The effect of increasing $N$ on the ratio $b_{2} / a_{1}$ is quite similar.

The ratio $a_{2} / a_{1}$ is identically equal to unity for scattering by a single sphere. Therefore, the rapidly growing deviation of this ratio from $100 \%$ for $N \geq 5$ in Fig. 9 can again be interpreted in RT terms as a direct consequence of the strengthened depolarizing effect of "multiple scattering" [7]. Analogously, $a_{3}(\Theta) \equiv a_{4}(\Theta)$ and $a_{3}\left(180^{\circ}\right) / a_{1}\left(180^{\circ}\right)=-1$ for scattering by a single spherically symmetric particle, but "multiple scattering" in particle groups with $N \geq 5$ causes an increasingly significant violation of these equalities.

Perhaps an even more convincing demonstration of the onset of the CB effect is provided by Fig. 11a. Indeed, the $\left(a_{1}+a_{4}\right) / 2$ curve for an isolated particle shows no backscattering enhancement at all, whereas those for the multi-particle volumes show pronounced backscattering peaks. The polarization curve for $N=1$ 
a
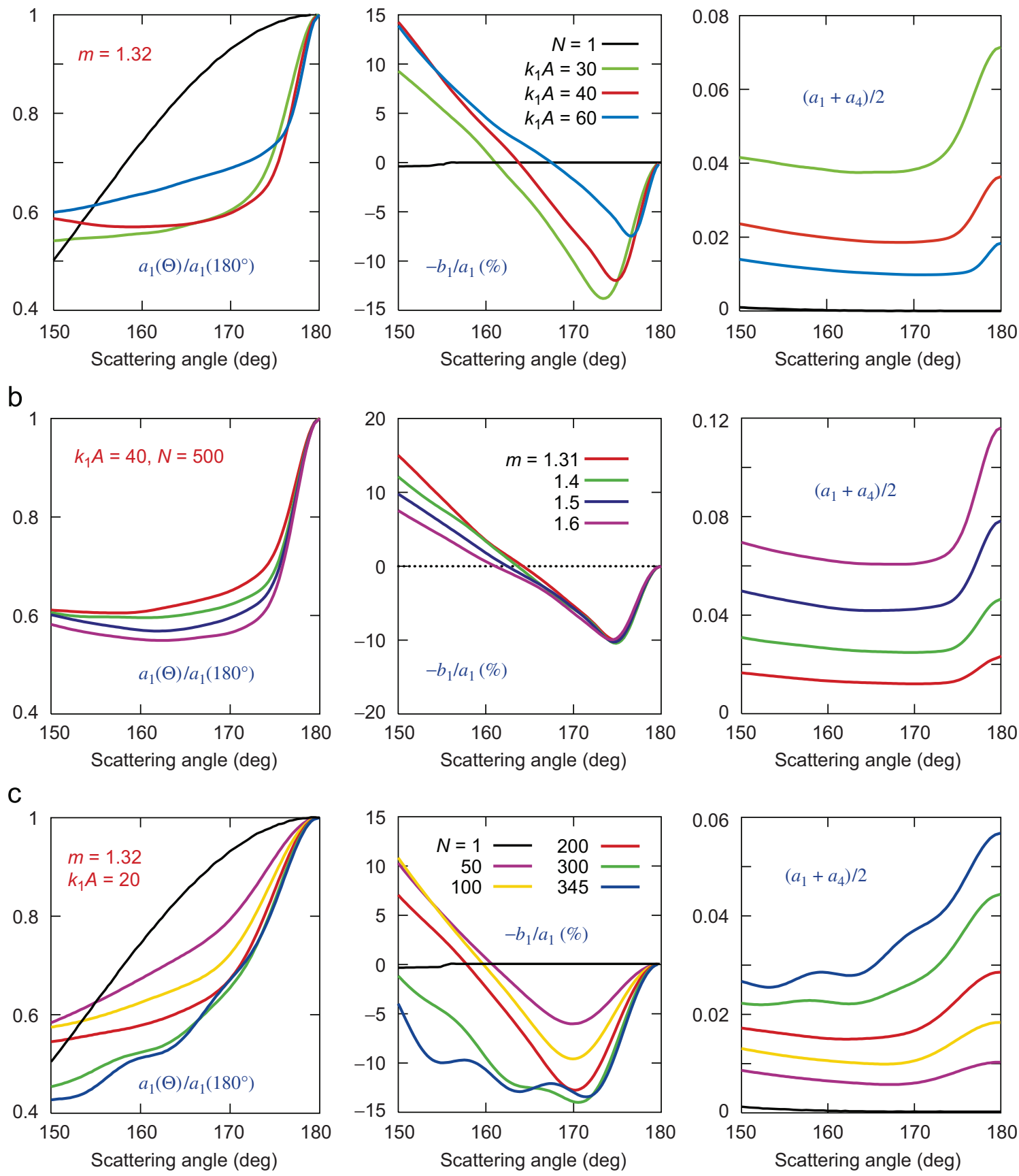

Fig. 11. (a) Scattering characteristics of a spherical scattering volume randomly filled with $N=800$ spherical particles. The particle refractive index and size parameter are fixed at $m=1.31$ and $k_{1} a=2$, respectively. The size parameter of the volume $k_{1} A$ varies from 30 to 60 . For comparison, the black curves depict the respective single-particle characteristics. (b) As in panel a, but for fixed $k_{1} A=40$ and $N=500$. The particle refractive index varies from $m=1.31$ to 1.6. The respective particle size-parameter values are indicated in Fig. 4e. (c) As in panel a, but for fixed $m=1.32$ and $k_{1} A=20$. The number of particles $N$ increases from 1 to 345 , resulting in packing densities (defined as if the volume were infinite [79]) up to 47\%.

reveals uniformly neutral polarization, whereas those for the multi-particle volumes exhibit a narrow asymmetric negative-polarization minimum at backscattering angles. This feature is predicted by the low-density theory of $\mathrm{CB}$ for Rayleigh constituent particles and is called the polarization opposition effect (POE) $[81,82]$. Importantly the angular widths of all the backscattering peaks and the angles of minimal polarization in Fig. 11 a scale as $1 / k_{1} A$ which is consistent with their interference $C B$ origin [cf. Eq. (31)]. 

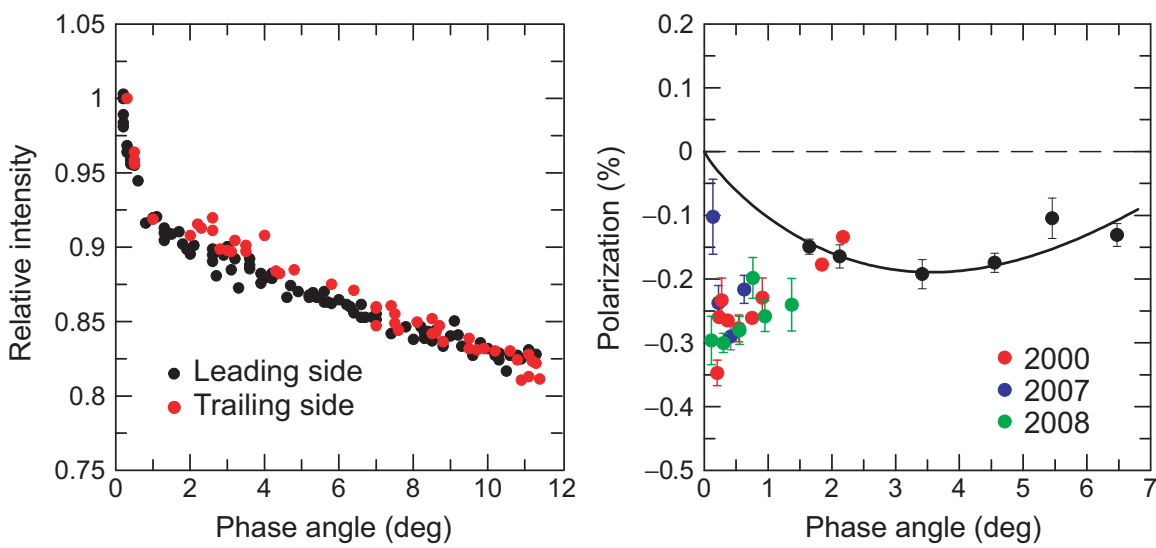

Fig. 12. Brightness [92] and polarization [78,97,100] opposition effects observed for Europa. The phase angle $\alpha$ is defined as the angle between the source of light and the detector as viewed from the scattering object and is given by $180^{\circ}-\Theta$ (see Fig. 10).

Fig. 11b demonstrates that the angular widths of these backscattering features are virtually independent of the particle refractive index, thereby further corroborating their $\mathrm{CB}$ nature. Furthermore, all four polarization curves exhibit the POE despite the complete absence of this feature in the respective single-particle polarization curves (Fig. 4e).

The effect of increasing the number of particles $N$ in a volume of DRM can be expected to be twofold. On one hand, it facilitates "multiple scattering" and thereby enhances the classical manifestations of RT and CB. On the other hand, it leads to increased packing density and can eventually cause changes in the scattering patterns not implied by the low-packing-density theories of RT and CB $[83,84]$. One should, therefore, expect that the RT and $\mathrm{CB}$ interpretation of numerically-exact $T$-matrix data must ultimately become inadequate when the particle packing density becomes exceedingly large. Fig. 11c shows that this is indeed the case: the curves for $N=300$ and 345 develop high-frequency interference ripple typical of a compact particle with a size parameter $k_{1} A=20$ (cf. Fig. 3 and right-hand panels of Figs. 6a and b) which, of course, is not predicted by the RT and CB theories. Nevertheless, the direct solutions of the MMEs do demonstrate that the classical predictions of these low-density theories can survive (at least in a semiquantitative sense) volume packing densities typical of particle suspensions and particulate surfaces [78,79].

This result raises an interesting question [85] of whether one can legitimately attribute to the effect of $\mathrm{CB}$ certain results of photometric and polarimetric observations of particulate planetary surfaces at small phase angles [86-101] such as those depicted in Fig. 12. In principle, this attribution is as unnecessary as the introduction of the mathematical concept of $C B$ in the first place. What matters is whether the results of photopolarimetric astronomical observations can be accurately reproduced by a direct solution of the MMEs for a realistic model of the scattering medium. However, attempting this is still impracticable, which makes it tempting to interpret the results of observations using idealized concepts such as RT and CB. Fortunately, the photometric and polarimetric data for Europa (Fig. 12) and several other high-albedo solar system objects [85] are quite unique in that they reveal both the photometric opposition effect and the POE of nearly equal angular widths and with angular profiles consistent with the exact solutions of the MMEs (Figs. 11a and b). No other honest theory of electromagnetic scattering has been demonstrated to yield both effects with their very specific traits simultaneously. Therefore, it appears to be rather safe to conclude that both opposition effects can be attributed, at least qualitatively, to coherent backscattering of sunlight by planetary surfaces composed of microscopic regolith particles $[78,85]$.

\section{Scalar approximation}

The honest microphysical derivation of the theories of RT and CB helps expose and dispel such fundamental a misconception as the scalar approximation (SA). It is widely believed that if one is interested only in the intensity of the scattered light but not in its polarization state then polarization can be ignored altogether, and the vector theories of RT and $\mathrm{CB}$ can be replaced by their "scalar" analogues. However, the SA is a phenomenological approach in that it does not follow directly from the MMEs. Therefore, the numerical accuracy of the SA must be carefully and extensively examined by comparing "scalar" results with those obtained using the honest vector versions of the RT and CB theories. Obviously, the SA should be expected to fail when the incident light is polarized. The inherently vector nature of electromagnetic radiation can cause significant errors in "scalar" computations even when the incident light is unpolarized, especially in computations of CB (e.g., [7,102,103] and references therein).

\section{Energy conservation}

Another widespread misconception is that the RTE is a direct consequence of applying trivial energy-balance considerations to an "elementary volume element" of a 
turbid medium. The fallaciousness of this belief becomes immediately obvious as soon as one recalls that the RTE does not describe per se specific manifestations of $C B$, including the angular profile of the backscattering intensity peak. Consider, for example, a semi-infinite cloud layer composed of nonabsorbing particles. The exact solution of the RTE suggests that the amount of energy diffusely reflected by this layer is identically equal to the amount of incident energy. However, adding the missing energy contained in the $\mathrm{CB}$ intensity peak breaks the energy conservation law: the amount of energy exiting the nonabsorbing cloud layer exceeds the amount of energy entering it.

In fact, it is a pure accident that summing up only the ladder diagrams (Section 6) results in an equation that by itself satisfies the energy conservation law. This implies that the result of summing up the energy contributions of all the other types of diagrams, including the cyclical diagrams, must be equal to zero. The amount of energy contained in the $\mathrm{CB}$ intensity peak is very small in the case of a sparse scattering object with dimensions much greater than the wavelength, e.g., a typical cloud of liquid water droplets or ice crystals. Indeed, for such a medium the angular width of the $\mathrm{CB}$ peak is inversely proportional to the product of the wave number $k_{1}$ and the so-called transport mean free path $l_{\mathrm{tr}}$ [58] and is negligibly small. However, the application of the low-density theories of RT and $\mathrm{CB}$ to densely packed scattering media may result in a noticeable violation of the energy conservation law.

A direct numerically-exact solution of the MMEs is, of course, free of this problem. Indeed, the single-scattering albedo $\varpi$ of any of the particulate volumes used to make Figs. 9 and 11 was found to be precisely unity irrespective of the particle packing density. This means that the amount of energy entering a particulate volume is identical to the amount of energy exiting it. Let us see, however, what happens when one tries to solve the scattering problem by replacing the original FLEs with their far-field version (23) and (24). This is an instructive exercise since the FLEs form the very basis of the microphysical theories of RT and CB.

To do such computations, we use the same superposition $T$-matrix code [49] but modify the coefficients appearing in a certain translation-addition theorem for vector spherical wave functions. Specifically we artificially replace the Hankel functions of the first kind $h_{n}^{(1)}\left(k_{1} R_{i j}\right)$, where $R_{i j}$ is the distance between the origins of particles $i$ and $j$ (Fig. 8), with their asymptotic far-field limits $(-\mathrm{i})^{n+1} \exp \left(\mathrm{i} k_{1} R_{i j}\right)\left(k_{1} R_{i j}\right)^{-1}$. We have found that such computations for $k_{1} A=30, k_{1} a=2$, and $m=1.31$ result in $\varpi=1.0035$ for $N=10$ and $\varpi=1.0900$ for $N=100$. Similar computations for $k_{1} A=40, k_{1} a=4$, and $m=1.32$ yield $\varpi=1.0004$ for $N=2, \varpi=1.0157$ for $N=5, \varpi=1.2344$ for $N=10, \varpi=1.2970$ for $N=20$, and $\varpi=1.4903$ for $N=40$. Obviously, these numbers demonstrate a significant violation of the energy conservation law in the form of surplus scattered energy.

Since the modified superposition $T$-matrix code still includes all types of multi-particle wave paths rather than only the self-avoiding paths (Section 6), one may hypothesize that the artificial replacement of near-field with far-field interactions exaggerates the contribution of wave paths going through a particle more than once. This result demonstrates the importance of ignoring non-selfavoiding paths in addition to ignoring CB in the microphysical derivation of the energy-conservation-compliant RTE.

\section{Particles as individual scatterers}

One of the most fundamental misconceptions in the phenomenological RTT is that each particle in a sparse multi-particle group is an individual scatterer characterized by its own extinction, scattering, and absorption cross sections, scattering matrix, and extinction matrix. In other words, it is assumed that each particle possesses an individual set of optical characteristics quantifying the single-scattering transformation of the intensity (or the Stokes parameters) rather than that of the electromagnetic field. It is then postulated that the above individual characteristics of particles contained in a "differential volume element" of the DRM must be added to yield those of the volume element. Finally, the resulting cumulative characteristics enter the RTE.

It is then admitted that two factors can "modify" the individual-particle optical characteristics:

- "effects of packing density" in a densely packed DRM;

- non-zero absorptivity of the host medium surrounding the particles.

Numerous publications have been devoted to extensive discussions of how these modifications might work and how they should be accounted for in an appropriately modified RTE.

This approach to RT is fundamentally wrong. As we have seen in Section 5, the basic individual scattering characteristic of particle $i$ in a multi-particle group is its dyadic transition operator $\hat{T}_{i}$. If the particles in the group are widely separated then the role of the unique scattering identifier of a particle is assumed by its farfield scattering dyadic $\hat{A}_{i}$. Both dyadics quantify the transformation of the electromagnetic field rather than that of the intensity. As a consequence of statistical averaging required to derive the RTE from the far-field FLEs, the individual particles forming the DRM effectively disappear. Instead, the resulting RTE is found to contain phase and extinction matrices ensemble-averaged over all the particles contained in the DRM. Thus, under no circumstances can a particle in the DRM be characterized by individual optical cross sections and phase and extinction matrices.

In the case of a densely-packed DRM the original FLEs cannot be replaced by the far-field FLEs, the singleparticle dyadic transition operators $\hat{T}_{i}$ cannot be replaced by the corresponding far-field scattering dyadics $\hat{A}_{i}$, the classical RTE cannot be derived, and individual-particle or even ensemble-averaged extinction and phase matrices never appear. Thus, there are no packing-density effects allegedly modifying individual-particle optical characteristics. 
In the case of an absorbing host medium, the focal point of the discussions was the likely effect of absorption on the conventional scattering cross section, single-scattering albedo, and phase function of an isolated particle and a differential volume element. However, the application of the microphysical approach to the derivation of formulas describing actual optical observables shows that these quantities cannot even be defined in the case of electromagnetic scattering by an isolated particle embedded in an absorbing medium [104-106]. Instead, one can define the extinction and phase matrices of an isolated particle as well as derive the generalized RTE for a sparse DRM in much the same way as it is done in the case of a nonabsorbing host [106-108]. In particular, Eq. (32) is replaced by

$$
\begin{aligned}
\hat{\mathbf{q}} \cdot \nabla \tilde{\mathbf{I}}(\mathbf{r}, \hat{\mathbf{q}})= & -2 k_{1}^{\prime \prime} \tilde{\mathbf{I}}(\mathbf{r}, \hat{\mathbf{q}})-n_{0}\langle\mathbf{K}(\hat{\mathbf{q}})\rangle_{\xi} \tilde{\mathbf{I}}(\mathbf{r}, \hat{\mathbf{q}}) \\
& +n_{0} \int_{4 \pi} \mathrm{d} \hat{\mathbf{q}}^{\prime}\left\langle\mathbf{Z}\left(\hat{\mathbf{q}}, \hat{\mathbf{q}}^{\prime}\right)\right\rangle_{\xi} \tilde{\mathbf{I}}\left(\mathbf{r}, \hat{\mathbf{q}}^{\prime}\right),
\end{aligned}
$$

where $k_{1}^{\prime \prime}$ is the imaginary part of the complex-valued wave number of the host medium.

\section{Coherent field and effective dielectric constant}

Finally, let us discuss the widespread misconception according to which the so-called coherent field $\mathbf{E}_{\mathrm{c}}(\mathbf{r})$ is a real electromagnetic wave which propagates in a DRM and is subject to exponential attenuation owing to a nonzero imaginary part of the so-called dyadic propagation constant $\overleftrightarrow{\kappa}$. The source of this misinterpretation is the following differential equation for the coherent field [7]:

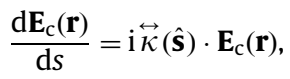

where the differential path-length $\mathrm{d} s$ is measured along the unit vector $\hat{\mathbf{s}}$ and the dyadic propagation constant for the propagation direction $\hat{\mathbf{s}}$ is given by

$\overleftrightarrow{\kappa}(\hat{\mathbf{s}})=k_{1} \overleftrightarrow{I}+\frac{2 \pi n_{0}}{k_{1}}\langle\overleftrightarrow{A}(\hat{\mathbf{s}}, \hat{\mathbf{s}})\rangle \xi$

An equation similar to Eq. (38) would describe, indeed, the amplitude of a time-harmonic plane electromagnetic wave as it propagates in a homogeneous (and generally anisotropic) absorbing medium. Therefore, it is often claimed, by analogy, that the coherent field in a DRM is an exponentially attenuated plane electromagnetic wave, $\overleftrightarrow{\kappa}(\hat{\mathbf{s}})$ is given by a standard expression in terms of the "effective" dyadic optical constants of the DRM, and the non-zero imaginary part of the dyadic propagation constant is caused by the presence of the particles. Based on this misinterpretation, it was postulated [109] that the effective dyadic optical constants of a DRM must satisfy the well-known Kramers-Kronig relations valid for a homogeneous medium. From this premise, Purcell [109] derived the sum rule for the extinction cross section discussed earlier in Section 3.

It should be recalled, however, that the incident plane electromagnetic wave is not attenuated exponentially inside the DRM but rather remains unchanged, as it should be with any incident field by definition [see Eq. (25)]. What is attenuated exponentially according to the dyadic propa- gation constant with a non-zero imaginary part is the timeindependent coherent field $\mathbf{E}_{\mathrm{c}}(\mathbf{r})$ obtained by

- factorizing the total electric field inside the medium as $\mathbf{E}_{0}(\mathbf{r}, t) \exp (-\mathrm{i} \omega t)$, where the electric field amplitude $\mathbf{E}_{0}(\mathbf{r}, t)$ is a "slowly-varying" function of time provided that significant changes in particle positions occur over time intervals much longer than the period of timeharmonic oscillations $2 \pi / \omega$;

- artificially neglecting the time-harmonic factor $\exp (-\mathrm{i} \omega t)$, because otherwise averaging over time would give a zero net result:

$\frac{1}{T} \int_{t}^{t+T} \mathrm{~d} t^{\prime} \exp \left(-\mathrm{i} \omega t^{\prime}\right)_{T \gg}=\underset{2 \pi / \omega}{=} 0$

- expressing the random amplitude $\mathbf{E}_{0}(\mathbf{r}, t)$ as a sum of the time-independent coherent (average) field $\mathbf{E}_{\mathrm{c}}(\mathbf{r})$ and a fluctuating field $\mathbf{E}_{\mathrm{f}}(\mathbf{r}, t)$ caused by random changes in particle positions;

- calculating $\mathbf{E}_{\mathrm{c}}(\mathbf{r})$ as the average of $\mathbf{E}_{0}(\mathbf{r}, t)$ over a time interval long enough to establish full ergodcity of the DRM.

We thus see that the coherent field is an artificial mathematical construction rather than an actual timedependent electromagnetic field. In particular, it is not a time-harmonic plane electromagnetic wave. The only reason to consider this purely mathematical quantity in the first place is that it turns out to be useful in the microphysical derivation of the RTE [7].

Thus, we must conclude that the effective dyadic optical constants of a DRM are not physical dyadic optical constants appearing in expressions for actual time-dependent physical fields in a homogeneous medium. This conclusion shows that there is no reason whatsoever to state that the former satisfy the Kramers-Kronig relations and negates the derivation of the sum rule in [109].

\section{Concluding remarks}

The very tone of Keller's classification and terminology [1] suggests that it may have been introduced with an implicit goal to eventually drive phenomenological theories out of circulation. For example, in another milestone treatise on electromagnetic scattering by random media published six years after Keller's paper, Frisch [110] states that even if completely dishonest methods "have produced many interesting results in the past, they are now obsolete, for using dishonest methods is like gambling: one does not know in advance whether the results will be valid or not." But even now, almost 50 years after the publication of Keller's paper, phenomenological methods are still widely used and are unlikely to get abandoned in the near future. The main reasons for that are, of course, the ever increasing complexity of electromagnetic scattering problems needing solution and the perennial inadequacy of available computer resources.

As we have mentioned above, the analytical simplicity of phenomenological approaches can sometimes yield useful physical insights. Furthermore, there are encouraging cases of phenomenological approaches paving the way to advanced 
microphysical techniques. Among the most instructive examples is the transformation of the discrete dipole approximation from a phenomenological substitution of a scatterer by a set of point dipoles [111] into a rigorous corollary of the MMEs (see [26,112] and references therein).

Still it is critically important to be aware of the need to examine carefully the origin of any phenomenological approach and analyze thoroughly its likely errors. Quite often a phenomenological theory is the source of misconceptions and misinterpretations. There are phenomenological theories which appear to be enticingly simple and attractive and suggest an easy way for an observational astronomer or a remote-sensing scientist to publish a paper containing both the results of observations and their "theoretical interpretation"-an unjust but standard requirement in some journals. However, the customers of such populist "theories" (e.g., the notorious "Hapke model") should realize that their papers can become casualties of a thoroughly unphysical analysis tool (see, e.g., [3,113] and references therein).

Finally, it is important to remember that even honest mathematical concepts such as the concepts of RT and CB have their limitations and, in the final analysis, are unnecessary. The range of quantitative applicability of these concepts in situations violating the basic assumptions used in their derivations needs to be established by invoking the results of controlled laboratory experiments and/or numerically-exact computer solutions of the MMEs. There is no doubt that the ever increasing efficiency of computers will enable one to explore progressively sophisticated scattering models with direct solvers of the MMEs and will eventually provide the ultimate theoretical tool for the interpretation of remotesensing observations, thereby rendering the idealized concepts of CB and RT unnecessary.

\section{Acknowledgements}

We thank Matthew Berg, Anthony Davis, Steven Hill, Joop Hovenier, Michael Kahnert, Nikolai Khlebtsov, Andrew Lacis, Dan Mackowski, Pinar Mengüç, Gorden Videen, Ping Yang, and Maxim Yurkin for numerous useful discussions. Maxim Yurkin and two anonymous reviewers provided very helpful, constructive, and encouraging comments on the original manuscript. This project was sponsored by the NASA Radiation Sciences Program managed by Hal Maring.

\section{Appendix A. List of acronyms}

CB
DRM
FLES
GOM
GTD
MMEs
POE
QED
RT
RTE
RTT
SA
VIE

coherent backscattering discrete random medium Foldy-Lax equations geometrical optics method geometrical theory of diffraction macroscopic Maxwell equations polarization opposition effect quantum electrodynamics radiative transfer radiative transfer equation radiative transfer theory scalar approximation volume integral equation

\section{References}

[1] Keller JB. Wave propagation in random media. Proc Symp Appl Math 1962;13:227-46.

[2] Dassios G, Kleinman R. Low frequency scattering. Oxford: Clarendon Press; 2000.

[3] Tishkovets VP, Mishchenko MI. Coherent backscattering: conceptions and misconceptions. JQSRT 2010;111:645-9.

[4] Borovikov VA, Kinber BY. Geometrical theory of diffraction. Herts, UK: IEE Press; 1994.

[5] James GL. Geometrical theory of diffraction for electromagnetic waves. Herts, UK: IET Press; 2007.

[6] Stratton JA. Electromagnetic theory. New York: McGraw-Hill; 1941.

[7] Mishchenko MI, Travis LD, Lacis AA. Multiple scattering of light by particles: radiative transfer and coherent backscattering. Cambridge, UK: Cambridge University Press; 2006.

[8] Mishchenko MI. Multiple scattering, radiative transfer, and weak localization in discrete random media: unified microphysical approach. Rev Geophys 2008;46:RG2003.

[9] Silver S, editor. Microwave antenna theory and design. New York: McGraw-Hill; 1949.

[10] Müller C. Foundations of the mathematical theory of electromagnetic waves. Berlin: Springer; 1969.

[11] Akhiezer AI, Peletminskii SV. Methods of statistical physics. Oxford: Pergamon Press; 1981.

[12] Umezawa $\mathrm{H}$, Matsumoto $\mathrm{H}$, Tachiki M. Thermo field dynamics and condensed states. Amsterdam: North-Holland; 1982.

[13] Jackson JD. Classical electrodynamics. New York: Wiley; 1999.

[14] Van Bladel J. Electromagnetic fields. Piscataway, NJ: IEEE Press; 2007.

[15] Bohm D. Quantum theory. Englewood Cliffs, NJ: Prentice-Hall; 1951

[16] Kramers HA. Quantum mechanics. Amsterdam: North-Holland; 1957.

[17] Bogoliubov NN, Shirkov DV. Introduction to the theory of quantized fields. New York: Interscience; 1959.

[18] Power EA. Introductory quantum electrodynamics. London: Longmans; 1964.

[19] Akhiezer AI, Berestetskii VB. Quantum electrodynamics. New York: Wiley Interscience; 1965.

[20] Mandel L, Wolf E. Optical coherence and quantum optics. Cambridge, UK: Cambridge University Press; 1995.

[21] Meystre P, Sargent III M. Elements of quantum optics. Berlin: Springer; 1999.

[22] Kidd R, Ardini J, Anton A. Evolution of the modern photon. Am J Phys 1989;57:27-35.

[23] Scully MO, Sargent III M. The concept of the photon. Phys Today 1972;3:38-47.

[24] Lukš A, Peřinová V. Quantum aspects of light propagation. Berlin: Springer; 2009.

[25] Mishchenko MI. Gustav Mie and the fundamental concept of electromagnetic scattering: a perspective. JQSRT 2009;110: 1210-22.

[26] Yurkin MA, Hoekstra AG. The discrete dipole approximation: an overview and recent developments. JQSRT 2007;106:558-89.

[27] Bohren CF, Huffman DR. Absorption and scattering of light by small particles. New York: Wiley; 1983.

[28] Mishchenko MI. Broadband electromagnetic scattering by particles. J Opt Soc Am A 2008;25:2893-5.

[29] Liou KN, Takano Y, Yang P. Light scattering and radiative transfer in ice crystal clouds: applications to climate research. In: Mishchenko MI, Hovenier JW, Travis LD, editors. Light scattering by nonspherical particles. San Diego: Academic Press; 2000. p. 417-49.

[30] Yang P, Liou KN. Light scattering and absorption by nonspherical ice crystals. Light Scattering Rev 2006;1:31-71.

[31] van de Hulst HC. Light scattering by small particles. New York: Dover; 1981.

[32] Newton RG. Scattering theory of waves and particles. New York: Springer; 1982

[33] Nussenzveig HM. Diffraction effects in semiclassical scattering. Cambridge, UK: Cambridge University Press; 1992.

[34] Grandy WT Jr. Scattering of waves from large spheres. Cambridge, UK: Cambridge University Press; 2000.

[35] Adam JA. The mathematical physics of rainbows and glories. Phys Rep 2002;356:229-365.

[36] Mishchenko MI, Travis LD, Lacis AA. Scattering, absorption, and emission of light by small particles. Cambridge, UK: Cambridge University Press; $2002<$ http://www.giss.nasa.gov/ crmim/books html $>$. 
[37] Kahnert FM. Numerical methods in electromagnetic scattering theory. JQSRT 2003;79/80:775-824.

[38] Wriedt T. Light scattering theories and computer codes. JQSRT 2009;110:833-43.

[39] Muinonen K. Scattering of light by crystals: a modified Kirchhoff approximation. Appl Opt 1989;28:3044-50.

[40] Mazeron P, Muller S. Light scattering by ellipsoids in a physical optics approximation. Appl Opt 1996;35:3726-35.

[41] Hesse E, Mc Call DS, Ulanowski Z, Stopford C, Kaye PH. Application of RTDF to particles with curved surfaces. JQSRT 2009;110: 1599-603.

[42] Liou K-N, Hansen JE. Intensity and polarization for single scattering by polydisperse spheres: a comparison of ray optics and Mie theory. J Atmos Sci 1971;28:995-1004.

[43] Macke A, Mishchenko MI, Muinonen K, Carlson BE. Scattering of light by large nonspherical particles: ray tracing approximation versus $T$-matrix method. Opt Lett 1995;20:1934-6.

[44] Wielaard DJ, Mishchenko MI, Macke A, Carlson BE. Improved $T$-matrix computations for large, nonabsorbing and weakly absorbing nonspherical particles and comparison with geometrical-optics approximation. Appl Opt 1997;36:4305-13.

[45] Bi L, Yang P, Kattawar GW, Kahn R. Single-scattering properties of triaxial ellipsoidal particles for a size parameter range from the Rayleigh to geometric-optics regimes. Appl Opt 2009;48: 114-26.

[46] Bi L, Yang P, Kattawar GW, Kahn R. Modeling optical properties of mineral aerosol particles by using nonsymmetric hexahedra. Appl Opt 2010;49:334-42.

[47] Yurkin MA, Semyanov KA, Maltsev VP, Hoekstra AG. Discrimination of granulocyte subtypes from light scattering: theoretical analysis using a granulated sphere model. Opt Express 2007;15:16561-80.

[48] Hansen JE, Travis LD. Light scattering in planetary atmospheres. Space Sci Rev 1974;16:527-610.

[49] Mackowski DW, Mishchenko MI. Calculation of the $T$ matrix and the scattering matrix for ensembles of spheres. J Opt Soc Am A 1996;13:2266-78.

[50] Babenko VA, Astafyeva LG, Kuzmin VN. Electromagnetic scattering in disperse media: inhomogeneous and anisotropic particles. Chichester, UK: Praxis; 2003.

[51] Draine BT. The discrete dipole approximation for light scattering by irregular targets. In: Mishchenko MI, Hovenier JW, Travis LD, editors. Light scattering by nonspherical particles: theory, measurements, and applications. San Diego: Academic Press; 2000. p. 131-45

[52] Lommel E. Die Photometrie der diffusen Zurückwerfung. Sitzber Acad Wissensch München 1887;17:95-124.

[53] Chwolson O. Grundzüge einer mathematischen Theorie der inneren Diffusion des Lichtes. Bull l'Acad Impériale Sci St Pétersbourg 1889;33:221-56.

[54] Schuster A. Radiation through a foggy atmosphere. Astrophys J 1905;21:1-22.

[55] Apresyan LA, Kravtsov YuA. Radiation transfer. Statistical and wave aspects. Basel: Gordon and Breach; 1996.

[56] Mishchenko MI. Vector radiative transfer equation for arbitrarily shaped and arbitrarily oriented particles: a microphysical derivations from statistical electromagnetics. Appl Opt 2002;41: 7114-34.

[57] Mishchenko MI. Microphysical approach to polarized radiative transfer: extension to the case of an external observation point. Appl Opt 2003;42:4963-7.

[58] Barabanenkov YuN, Kravtsov YuA, Ozrin VD, Saichev AI. Enhanced backscattering in optics. Progr Opt 1991;29:65-197.

[59] Hansen JE, Hovenier JW. Interpretation of the polarization of Venus. J Atmos Sci 1974;31:1137-60.

[60] Wolf P, Maret G. Weak localization and coherent backscattering of photons in disordered media. Phys Rev Lett 1985;55:2696-9.

[61] Mishchenko MI. Polarization effects in weak localization of light: calculation of the copolarized and depolarized backscattering enhancement factors. Phys Rev B 1991;44:12597-600.

[62] Tishkovets VP, Mishchenko MI. Approximate calculation of coherent backscattering for semi-infinite discrete random media. JQSRT 2009;110:139-45.

[63] Yang P, Liou KN. Finite difference time domain method for light scattering by nonspherical and inhomogeneous particles. In: Mishchenko MI, Hovenier JW, Travis LD, editors. Light scattering by nonspherical particles: theory, measurements, and applications. San Diego: Academic Press; 2000. p. 173-221.

[64] Taflove A, Hagness SC. Computational electrodynamics: the finitedifference time-domain method. Boston: Artech House; 2000.
[65] Tseng SH, et al. Simulation of enhanced backscattering of light by numerically solving Maxwell's equations without heuristic approximations. Opt Express 2005;13:3666-72.

[66] Tseng SH, et al. Pseudospectral time domain simulations of multiple light scattering in three-dimensional macroscopic random media. Radio Sci 2006;41:RS4009.

[67] Mishchenko MI, Liu L. Weak localization of electromagnetic waves by densely packed many-particle groups: exact 3D results. JQSRT 2007;106:616-21.

[68] Mishchenko MI, Liu L, Mackowski DW, et al. Multiple scattering by random particulate media: exact 3D results. Opt Express 2007; $15: 2822-36$

[69] Mishchenko MI, Liu L, Videen G. Conditions of applicability of the single-scattering approximation. Opt Express 2007;15:7522-7.

[70] Mishchenko MI, Liu L, Hovenier JW. Effects of absorption on multiple scattering by random particulate media: exact results. Opt Express 2007;15:13182-7.

[71] Tseng SH, Huang B. Comparing Monte Carlo simulation and pseudospectral time-domain numerical solutions of Maxwell's equations of light scattering by a macroscopic random medium. Appl Phys Lett 2007;91:051114.

[72] Mackowski DW, Mishchenko MI. Prediction of thermal emission and exchange among neighboring wavelength-sized spheres. J Heat Transfer 2008;130:112702.

[73] Tseng SH. Optical characteristics of a cluster of closely-packed dielectric spheres. Opt Commun 2008;281:1986-90.

[74] Sukhov A, Haefner D, Dogariu A. Coupled dipole method for modeling optical properties of large-scale random media. Phys Rev E 2008;77:066709.

[75] Okada Y, Kokhanovsky AA. Light scattering and absorption by densely packed groups of spherical particles. JQSRT 2009;110: 902-17.

[76] Mishchenko MI, Liu L. Electromagnetic scattering by densely packed particulate ice at radar wavelengths: exact theoretical results and remote-sensing implications. Appl Opt 2009;48: 2421-6.

[77] Voit F, Schäfer J, Kienle A. Light scattering by multiple spheres: comparison between Maxwell theory and radiative-transfer-theory calculations. Opt Lett 2009;34:2593-5.

[78] Mishchenko MI, Dlugach JM, Liu L, et al. Direct solutions of the Maxwell equations explain opposition phenomena observed for high-albedo solar system objects. Astrophys J 2009;705: L118-22.

[79] Mishchenko MI, Dlugach JM, Liu L. Azimuthal asymmetry of the coherent backscattering cone: exact theoretical results. Phys Rev A 2009;80:053824

[80] Hovenier JW, van der Mee C, Domke H. Transfer of polarized light in planetary atmospheres-basic concepts and practical methods. Dordrecht, The Netherlands: Kluwer; 2004

[81] Mishchenko MI. On the nature of the polarization opposition effect exhibited by Saturn's rings. Astrophys J 1993;411:351-61.

[82] Mishchenko MI, Luck J-M, Nieuwenhuizen TM. Full angular profile of the coherent polarization opposition effect. J Opt Soc Am A 2000; 17:888-91

[83] Petrova E, Tishkovets V, Jockers K. Modeling of opposition effects with ensembles of clusters: interplay of various scattering mechanisms. Icarus 2007;188:233-45.

[84] Tishkovets VP. Light scattering by closely packed clusters: shielding of particles by each other in the near field. JQSRT 2008;109:266572.

[85] Mishchenko MI, Rosenbush VK, Kiselev NN. Weak localization of electromagnetic waves and opposition phenomena exhibited by high-albedo atmosphereless solar system objects. Appl Opt 2006;45:4459-63.

[86] Lyot B. Recherche sur la polarisation de la lumiere des planetes et de quelques substances terrestres. Ann Obs Meudon VIII 1929;8: $1-161$.

[87] Franklin FA, Cook AF. Optical properties of Saturn's rings. II. Twocolor phase curves of the two bright rings. Astron J 1965;70: 704-20.

[88] Johnson PE, Kemp JC, King R, Parker TE, Barbour MS. New results from optical polarimetry of Saturn rings. Nature 1980;283: 146-149.

[89] Brown RH, Cruikshank DP. The Uranian satellites: surface compositions and opposition brightness surges. Icarus 1983;55:83-92.

[90] Harris AW, Young JW, Contreiras L, Dockweiler T, Belkora L, Salo H, et al. Phase relations of high albedo asteroids: the unusual opposition brightening of 44 Nysa and 64 Angelina. Icarus 1989;81:365-74. 
[91] Goguen JO, Hammel HB, Brown RH. V photometry of Titania, Oberon and Triton. Icarus 1989;77:239-47.

[92] Thompson DT, Lockwood GW. Photoelectric photometry of Europa and Callisto 1976-1991. J Geophys Res 1992;97:14761-72.

[93] Rosenbush VK, Avramchuk VV, Rosenbush AE, Mishchenko MI. Polarization properties of the Galilean satellites of Jupiter: observations and preliminary analysis. Astrophys J 1997;487: 402-14.

[94] Helfenstein P, Currier N, Clark BE, Veverka J, Bell M, Sullivan R, et al. Galileo observations of Europa's opposition effect. Icarus 1998;135:41-63.

[95] Rosenbush VK, Kiselev NN, Jockers K, Korokhin VV, Shakhovskoj NM, Efimov YuS. Optical polarimetry of the Galilean satellites, Iapetus, and 64 Angelina near opposition. Kinem Phys Celest Bodies Suppl Ser 2000;3:227-30.

[96] Rosenbush VK, Kiselev NN, Shevchenko VG, Jockers K, Shakhovskoy NM, Efimov YuS. Polarization and brightness opposition effects for the E-type asteroid 64 Angelina. Icarus 2005;178: 222-34.

[97] Rosenbush VK, Kiselev NN. Polarization opposition effect for the Galilean satellites of Jupiter. Icarus 2005;179:490-6.

[98] Avramchuk VV, Rosenbush VK, Bul'ba TP. Photometric study of the major satellites of Uranus. Solar Syst Res 2007;41:186-202.

[99] Rosenbush V, Shevchenko V, Kiselev N, Sergeev A, Shakhovskoy N, Velichko F, et al. Polarization and brightness opposition effects for E-type asteroid 44 Nysa. Icarus 2009;201:655-65.

[100] Kiselev N, Rosenbush V, Velichko F, Zaitsev S. Polarimetry of the Galilean satellites and Jupiter near opposition. JQSRT 2009;110: 1713-8.

[101] Kolokolova L, Buratti B, Tishkovets V. Impact of coherent backscattering on the spectra of icy satellites of Saturn and the implications of its effects for remote sensing. Astrophys J 2010;711:L71-4.
[102] Lacis AA, Chowdhary J, Mishchenko MI, Cairns B. Modeling errors in diffuse-sky radiation: vector vs. scalar treatment. Geophys Res Lett 1998;25:135-8.

[103] Mishchenko MI, Dlugach JM. Accuracy of the scalar approximation in computations of diffuse and coherent backscattering by discrete random media. Phys Rev A 2008;78:063822.

[104] Bohren CF, Gilra DP. Extinction by a spherical particle in an absorbing medium. J Colloid Interface Sci 1979;72:215-21.

[105] Videen G, Sun W. Yet another look at light scattering from particles in absorbing media. Appl Opt 2003;42:6724-7.

[106] Mishchenko MI. Electromagnetic scattering by a fixed finite object embedded in an absorbing medium. Opt Express 2007;15: 13188-202.

[107] Mishchenko MI. Multiple scattering by particles embedded in an absorbing medium. 1. Foldy-Lax equations, order-of-scattering expansion, and coherent field. Opt Express 2008;16:2288-301.

[108] Mishchenko MI. Multiple scattering by particles embedded in an absorbing medium. 2. Radiative transfer equation. JQSRT 2008;109:2386-90.

[109] Purcell EM. On the absorption and emission of light by interstellar grains. Astrophys J 1969;158:433-40.

[110] Frisch U. Wave propagation in random media. In: Bharucha-Reid AT, editor. Probabilistic methods in applied mathematics. New York: Academic Press; 1968. p. 75-198.

[111] Purcell EM, Pennypacker CR. Scattering and absorption of light by nonspherical dielectric grains. Astrophys J 1973;186:705-14.

[112] Yurkin MA, Maltsev VP, Hoekstra AG. Convergence of the discrete dipole approximation. I. Theoretical analysis. J Opt Soc Am A 2006;23:2578-91.

[113] Mishchenko MI, Macke A. Asymmetry parameters of the phase function for isolated and densely packed spherical particles with multiple internal inclusions in the geometric optics limit. JQSRT 1997;57:767-94. 